\title{
A GENERAL VIEW OF REFLEXIVITY
}

\author{
DON HADWIN
}

\begin{abstract}
Various concepts of reflexivity for an algebra or linear space of operators have been studied by operator theorists and algebraists. This paper contains a very general version of reflexivity based on dual pairs of vector spaces over a Hausdorff field. The special cases include topological, algebraic and approximate reflexivity. In addition general versions of hyperreflexivity and direct integrals are introduced. We prove general versions of many known (and some new) theorems, often with simpler proofs.
\end{abstract}

\section{INTRODUCTION}

The purpose of this paper is to unify some of the recent results on reflexivity for algebras and linear spaces of operators on Hilbert space as well as the algebraic and asymptotic analogues of these notions.

We first define a notion of reflexivity that contains the usual topological, approximate, and algebraic versions of reflexivity as special cases. We also define a notion of hyperreflexivity and of direct integrals in our general setting.

In this general setting we prove analogues of results of Azoff, Fong and Gilfeather [AFG], Loginov and Shulman [LS], Larson [L1, L2], Arveson [Ar2], Kraus and Larson [KL], Olin and thomson [OT], and Hadwin and Nordgren [HN1, HN2, HN3]. We also prove general results concerning hyperreflexive graphs and hyperreflexive direct integrals that yield new results even in the Hilbert space setting. Much of the remainder of the paper is spent interpreting and applying our results in various special cases to obtain several new results.

An important aspect of this generalized notion of reflexivity is that the proofs of the aforementioned results are never more difficult, and often easier and more transparent, in the more general setting.

At the end of the paper we discuss other versions of reflexivity that are not special cases of our generalized version. We also discuss briefly a generalized notion of transitivity.

One interesting, but relatively unimportant, aspect of our generalized notion of reflexivity is that it connects the notion of "reflexive algebra of operators" with the classical notion of "reflexive Banach space" (see the example below).

It is hoped that his general notion of reflexivity will increase the interplay between operator theory and general Banach space theory. In addition the general

Received by the editors August 26, 1992 and, in revised form June 25, 1993.

1991 Mathematics Subject Classification. Primary 46A19, 47D15; Secondary 46S10. 
setting provides a framework in which conjectures can be tested and counterexamples can be constructed.

\section{ReFLEXIVITY}

Suppose that $F$ is a topological field with a Hausdorff topology, e.g., $F$ could be a subfield of the complex numbers with the usual topology, or $F$ could be an arbitrary field with the discrete topology.

Next suppose that $X$ is a vector space over $F$ and $Y$ is a vector space of linear maps from $X$ to $F$ that separates the points of $X$ (i.e., $\bigcap\{\operatorname{ker} f: f \in$ $Y\}=\{0\})$. We call such a pair $(X, Y)$ a dual pair over $F$. We define the $\sigma(X, Y)$-topology on $X$ to be the smallest topology on $X$ that makes all of the maps in $Y$ continuous on $X$. Since we can view $X$ as a vector space of (evaluation) functionals on $Y$, we can define the $\sigma(Y, X)$-topology on $Y$ to be the smallest topology for which the map $f \mapsto f(x)$ is continuous on $Y$ for each $x$ in $X$.

If $A \subset X$ and $B \subset Y$, we define $A^{\perp}=\{f \in Y: f \mid A=0\}$ and $B_{\perp}=$ $\bigcap\{\operatorname{ker}(f): f \in B\}$. If $D$ is a subset of either $X$ or $Y$, then $\operatorname{sp}(D)$ denotes the linear span of $D$, and $\overline{\mathrm{sp}}(D)$ denotes the closure of $\operatorname{sp}(D)$ in either the $\sigma(X, Y)$ or $\sigma(Y, X)$ topology. The following familiar results from the duality theory of real or complex topological vector spaces hold in this more general setting. For completeness we include the simple proof.

Proposition 1.1. (1) A linear map $f: X \rightarrow F$ is $\sigma(X, Y)$-continuous if and only if $\operatorname{ker}(f)$ is $\sigma(X, Y)$-closed. $Y$.

(2) A linear map from $X$ to $F$ is $\sigma(X, Y)$-continuous if and only if it is in

(3) If $M$ is a linear subspace of $X$ and $g: M \rightarrow F$ is linear and $\sigma(X, Y)$ continuous, then there is an $f$ in $Y$ such that $f \mid M=g$.

(4) If $A \subset X$, then $\overline{\operatorname{sp}}(A)=\left(A^{\perp}\right)_{\perp}$.

(5) If $B \subset Y$, then $\overline{\mathrm{sp}}(B)=\left(B_{\perp}\right)^{\perp}$.

Proof. (1), (2). Suppose $f: X \rightarrow F$ is linear. Clearly if $f$ is $\sigma(X, Y)$ continuous, then $\operatorname{ker}(f)$ is closed. It is also evident that if $f \in Y$, then $f$ is $\sigma(X, Y)$-continuous. Suppose now that $\operatorname{ker}(f)$ is $\sigma(X, Y)$-closed; we will show that $f \in Y$. Assume that $f \neq 0$ and suppose $f(x) \neq 0$. Then there is a neighborhood $U$ of 0 in $F$ and functions $f_{1}, f_{2}, \ldots, f_{n}$ in $Y$ such that $\operatorname{ker}(f) \cap\left\{z \in X: f_{i}(z)-f_{i}(x) \in U\right\}=\varnothing$. It follows that if $w \in \bigcap_{i} \operatorname{ker}\left(f_{i}\right)$ and $\lambda \in F$, then $\lambda w-x \notin \operatorname{ker}(f)$, which implies $f(w)=0$. Since $\bigcap_{i} \operatorname{ker}\left(f_{i}\right) \subset$ $\operatorname{ker}(f)$, we conclude $f \in \operatorname{sp}\left\{f_{1}, f_{2}, \ldots, f_{n}\right\} \subset Y$.

(3) This follows from (2) and the fact that the relative $\sigma(X, Y)$-topology on $M$ is the $\sigma(M,\{f \mid M: f \in Y\})$-topology.

(4) Suppose $x \in X$ and $x \notin \overline{\mathrm{sp}}(A)$. Let $M=\operatorname{sp}(\{x\} \cup \overline{\mathrm{sp}}(A))$, and define a linear map $g: M \rightarrow F$ so that $g \mid \overline{\mathbf{s p}}(A)=0$ and $g(x)=1$. Since $\operatorname{ker}(g)=$ $\overline{\mathrm{sp}}(A)$ is $\sigma(X, Y)$-closed in $M$, it follows from (3) that there is an $f$ in $A^{\perp}$ such that $f(x)=1$. This proves $\left(A^{\perp}\right)_{\perp} \subset \overline{\mathrm{sp}}(A)$. The reverse inclusion is obvious.

(5) This is the same as (4), especially if you are dyslexic.

We define $(X, Y, E)$ to be a reflexivity triple over $F$ if $(X, Y)$ is a dual pair over $F$ and $\phi \neq E \subset Y$ such that $E$ is closed under multiplication by 
scalars and $E_{\perp}=\{0\}$. If $\mathscr{S}$ is a linear subspace of $X$, we define $\operatorname{Ref}_{E}(\mathscr{S})=$ $\left(\mathscr{S}^{\perp} \cap E\right)_{\perp}$. We say that $\mathscr{S}$ is E-reflexive if $\mathscr{S}=\operatorname{Ref}_{E}(\mathscr{S})$.

Examples. 1. The prime motivating example of a reflexivity triple is the case in which $X$ is the set of operators on a Hilbert space $H, Y$ is the set of weakoperator-continuous linear functionals on $X$, and $E$ is the set of rank-one tensors, i.e., mappings of the form $T \mapsto(T f, g)$, where $f$ and $g$ are fixed vectors in $H$. Suppose $\mathscr{S}$ is the unital subalgebra of $B(H)$. Then Lat $\mathscr{S}$ is the set of invariant (closed linear) subspaces for $\mathscr{S}$, and $\operatorname{Alg}$ Lat $\mathscr{S}=\{T \in$ $B(H)$ : Lat $\mathscr{S} \subset$ Lat $T\}$. The algebra $\mathscr{S}$ is called reflexive if $\mathscr{S}=\operatorname{Alg} \mathrm{Lat} \mathscr{S}$. In this case, we have Alg Lat $\mathscr{S}=\operatorname{Ref}_{E}(\mathscr{S})$. Thus reflexivity and $E$-reflexivity coincide for unital subalgebras of $B(H)$.

2. Suppose $Y$ is a Banach space and $X$ is the normal dual $Y^{\#}$ of $Y$. Let $E=Y$. If $\mathscr{S} \subset X$, then $\operatorname{Ref}_{E}(\mathscr{S})=\left(\mathscr{S}^{\perp}\right)_{\perp}$ is the weak *-closed linear span of $\mathscr{S}$. It follows that $Y$ is a reflexive Banach space (i.e., $Y=Y^{\# \#)}$ if and only if every norm closed linear subspace of $X$ is $E$-reflexive. To avoid confusion of terminology, we shall refer to a Banach space $Y$ for which $Y=Y^{\# \#}$ as classically reflexive.

We say that $\mathscr{S}$ is hereditarily E-reflexive with respect to $Y$ if $\mathscr{S}$ and each of its $\sigma(X, Y)$-closed linear subspaces is $E$-reflexive. We say that a linear functional $f$ in $Y$ is E-elementary on $\mathscr{S}$ if there is an $e$ in $E$ such that $f-e \in \mathscr{S}^{\perp}$. We say that $\mathscr{S}$ is E-elementary (with respect to $Y$ ) if each $f$ in $Y$ is $E$-elementary on $\mathscr{S}$, i.e., each continuous linear functional can be represented on $\mathscr{S}$ as an element of $E$. If $n$ is a positive integer, then $E_{n}=E+E+\cdots+E$ ( $n$ summands). The first two parts of the following theorem are generalizations of results of D. Larson [L1, L2], the third generalizes a result of A. Loginov and V. Shulman [LS], and the fourth generalizes a result in [RR, Theorem 7.1].

Theorem 1.2. Suppose $(X, Y, E)$ is a reflexivity triple over $F$, and $\mathscr{S}$ is a $\sigma(X, Y)$-closed linear subspace of $X$.

(1) The subspace $\mathscr{S}$ is E-reflexive if and only if $\mathscr{S}^{\perp}=\overline{\mathrm{sp}}\left(E \cap \mathscr{S}^{\perp}\right)$.

(2) If $\mathscr{S}$ is E-elementary and $\operatorname{sp}(E)=Y$, then $\mathscr{S}$ is $E_{3}$-reflexive.

(3) Suppose $\mathscr{S}$ is E-reflexive. Then $\mathscr{S}$ is hereditarily E-reflexive if and only if $\mathscr{S}$ is E-elementary.

(4) $\bigcap_{n \geq 1} \operatorname{Ref}_{E_{n}}(\mathscr{S})$ is the $\sigma(X, \operatorname{sp} E)$-closure of $\mathscr{S}$.

Proof. (1) It follows from Proposition 1.1 that $\mathscr{S}=\left(\mathscr{S}^{\perp}\right)_{\perp}=\left(E \cap \mathscr{S}^{\perp}\right)_{\perp}$ if and only if $\mathscr{S}^{\perp}=\overline{\mathrm{sp}}\left(E \cap \mathscr{S}^{\perp}\right)$.

(2) Suppose $f \in \mathscr{S}^{\perp}$. Since $\operatorname{sp} E=Y$, there is a smallest nonnegative integer $n$ such that $f$ can be written as $f=g+h_{1}+h_{2}+\cdots+h_{n}$, with $g$ in $\overline{\mathrm{sp}}\left(E_{3} \cap \mathscr{S}^{\perp}\right)$ and each $h_{i}$ in $E$. Assume via contradiction that $n \neq 0$. Then $n>3$, because $h_{1}+h_{2}+\cdots+h_{n}=f-g \in \mathscr{S}^{\perp}$. Since $\mathscr{S}$ is $E$-elementary, there is an $h$ in $E$ such that $h_{1}+h_{2}-h \in \mathscr{S}^{\perp}$. Hence $e=g+\left(h_{1}+h_{2}-h\right) \in$ $\overline{\mathrm{sp}}\left(E_{3} \cap \mathscr{S}^{\perp}\right)$, and $f=e+h+h_{3}+\cdots+h_{n}$, which violates the minimality of $n$. Thus $\mathscr{S}$ is $E_{3}$-reflexive.

(3) Suppose that $\mathscr{S}$ is hereditarily $E$-reflexive and suppose $f \in Y$. Then $\mathscr{S} \cap \operatorname{ker}(f)=\operatorname{ker}(f \mid \mathscr{S})$ is $E$-reflexive. Suppose $f \mid \mathscr{S} \neq 0$ and choose an $x$ in $\mathscr{S}$ such that $f(x) \neq 0$. Since $\operatorname{ker}(f \mid \mathscr{S})$ is $E$-reflexive, there is an $e$ in $E$ such that $\operatorname{ker}(f \mid \mathscr{S}) \subset \operatorname{ker}(e \mid \mathscr{S})$, and $e(x) \neq 0$. Since $E$ is closed under 
scalar multiplication, we can assume $e(x)=f(x)$, from which it follows that $f|\mathscr{S}=e| \mathscr{S}$, i.e., $f-e \in \mathscr{S}^{\perp}$. Thus $\mathscr{S}$ is $E$-elementary with respect to $Y$.

Conversely, suppose that $\mathscr{S}$ is $E$-reflexive and $E$-elementary, and let $M$ be a $\sigma(X, Y)$-closed linear subspace of $\mathscr{S}$. If $f \in M^{\perp}$, choose $e$ in $E$ such that $f-e \in \mathscr{S}^{\perp}=\overline{\mathrm{sp}}\left(E \cap \mathscr{S}^{\perp}\right) \subset \overline{\mathrm{sp}}\left(E \cap M^{\perp}\right)$. Since $e \in E \cap M^{\perp}$ and $f=e+(f-e)$, it follows that $M^{\perp}=\overline{\mathrm{sp}}\left(E \cap M^{\perp}\right)$. Thus, by (1) above, $M$ is $E$-reflexive, which shows that $\mathscr{S}$ is hereditarily $E$-reflexive.

(4) Let $\mathscr{S}_{1}$ denote the $\sigma(X, \mathrm{sp} E)$-closure of $\mathscr{S}$. Clearly, $\bigcap_{n \geq 1} \operatorname{Ref}_{E_{n}}(\mathscr{S})$ contains $\mathscr{S}_{1}$. Conversely, if $x \notin \mathscr{S}_{1}$, then there is an $f \in \operatorname{sp} E=\bigcup_{n \geq 1} E_{n}$ such that $f \mid \mathscr{S}=0$ and $f(x) \neq 0$. Hence, for some $n$, we have $x \notin \operatorname{Ref}_{E_{n}}(\mathscr{S})$.

We next extend a theorem of D. Hadwin and E. Nordgren on reflexive graphs [HN3]. Suppose $\left(X_{i}, Y_{i}, E_{i}\right)$ are reflexivity triples for $i=1,2$. Then there is a natural duality between $X_{1} \times X_{2}$ and $Y_{1} \times Y_{2}$ given by $\left(f_{1}, f_{2}\right)\left(x_{1}, x_{2}\right)=$ $f_{1}\left(x_{1}\right)+f_{2}\left(x_{2}\right)$. In this way $\left(X_{1} \times X_{2}, Y_{1} \times Y_{2}, E_{1} \times E_{2}\right)$ is a reflexivity triple.

Theorem 1.3. Suppose $\left(X_{i}, Y_{i}, E_{i}\right)$ is a reflexivity triple for $i=1,2$. Let $\mathscr{S}$ be a hereditarily $E_{1}$-reflexive linear subspace of $X_{1}$, and suppose that $\rho: \mathscr{S} \rightarrow X_{2}$ is linear and $\sigma\left(X_{1}, Y_{1}\right)-\sigma\left(X_{2}, Y_{2}\right)$ continuous. Then $\operatorname{graph}(\rho)$ is a hereditarily $E_{1} \times E_{2}$-reflexive linear subspace of $X_{1} \times X_{2}$.

Proof. Let $E=E_{1} \times E_{2}$. Suppose $\left(x_{1}, x_{2}\right) \in \operatorname{Ref}_{E}(\operatorname{graph}(\rho))$. Clearly, $x_{1} \in$ $\operatorname{Ref}_{E_{1}}(\mathscr{S})$. We can assume that $x_{1}=0$; otherwise replace $\left(x_{1}, x_{2}\right)$ by $\left(x_{1}, x_{2}\right)$ $-\left(x_{1}, \rho\left(x_{1}\right)\right)$. We need to show that $x_{2}=0$. Suppose that $e_{2} \in E_{2}$. Since $\mathscr{S}$ is $E_{1}$-elementary (Theorem 1.2(3)), there is an $e_{1}$ in $E_{1}$ such that $e_{2} \circ \rho-e_{1} \in \mathscr{S}^{\perp}$. Hence $\left(-e_{1}, e_{2}\right) \in\left(E_{1} \times E_{2}\right) \cap \operatorname{graph}(\rho)^{\perp}$, which implies that $\left(-e_{1}, e_{2}\right)\left(0, x_{2}\right)=e_{2}\left(x_{2}\right)=0$. Since $e_{2}$ was arbitrary in $E_{2}$, we conclude $x_{2} \in\left(E_{2}\right)_{\perp}=\{0\}$. This proves that $\operatorname{graph}(\rho)$ is $E_{1} \times E_{2}$-reflexive.

To show that $\operatorname{graph}(\rho)$ is $E_{1} \times E_{2}$-elementary, suppose $(f, g) \in Y_{1} \times Y_{2}$. Since $\mathscr{S}$ is $E_{1}$-elementary, there is an $e$ in $E_{1}$ such that $(f+g \circ \rho)-e \in \mathscr{S}^{\perp}$. Hence $(f, g)-(e, 0) \in \operatorname{graph}(\rho)^{\perp}$.

Remark. The reflexivity of $\operatorname{graph}(\rho)$ in the preceding theorem requires only the assumption that $\mathscr{S}$ be $E$-reflexive, $\rho$ be linear, and that $\left\{e_{2} \in E_{2}: \exists e_{1} \in E_{1}\right.$ with $\left.\left(e_{1}-e_{2} \circ \rho\right) \mid \mathscr{S}=0\right\}_{\perp}=0$ in $X_{2}$. Note that the reflexivity of graph $(\rho)$ implies that $\operatorname{graph}(\rho)$ is $\sigma\left(X_{1} \times X_{2}, Y_{1} \times Y_{2}\right)$-closed, which in many cases implies that $\rho$ is $\sigma\left(X_{1}, Y_{1}\right)-\sigma\left(X_{2}, Y_{2}\right)$ continuous.

\section{HYPERREFLEXIVITY}

In this section we assume that the field $F$ is either the real or complex numbers. We also assume that $X$ and $Y$ are (real or complex) normed spaces, with $Y$ a subspace of the normed dual $X^{\prime}$ of $X$. With these assumptions we call $(X, Y, E)$ a normed reflexivity triple. Throughout this section let $\widetilde{E}=\{e \in$ $E:\|e\|=1\}$, and for any subset $B$ of $Y$, let $\overline{c o}(B)$ denote the $\sigma(Y, X)$-closed convex hull of $B$ in $Y$.

Suppose $\mathscr{S}$ is a linear subspace of $X$. Define the seminorm $d_{Y}(, \mathscr{S})$ on $X$ by $d_{Y}(x, \mathscr{S})=\sup \left\{|f(x)|: f \in \mathscr{S}^{\perp},\|f\|=1\right\}$. Note that in many applications $d_{Y}(x, \mathscr{S})$ is the distance from $x$ to $\mathscr{S}$. We define another seminorm $d_{E}(, \mathscr{S})$ on $X$ by

$$
d_{E}(x, \mathscr{S})=\sup \left\{|f(x)|: f \in \widetilde{E} \cap \mathscr{S}^{\perp}\right\} .
$$


It is clear that $d_{E}(x, \mathscr{S}) \leq d_{Y}(x, \mathscr{S})$ for each vector $x$ in $X$. Moreover, it is clear that $x \in \operatorname{Ref}_{E}(\mathscr{S})$ if and only if $d_{E}(x, \mathscr{S})=0$. We say that $\mathscr{S}$ is $E$-hyperreflexive if there is a nonnegative constant $K$ such that $d_{Y}(x, \mathscr{S}) \leq$ $K d_{E}(x, \mathscr{S})$ for every $x$ in $X$; the smallest such $K$ is called the constant of E-hyperreflexivity of $\mathscr{S}$ and is denoted by $K_{E}(\mathscr{S})$. Clearly, hyperreflexivity implies reflexivity.

The following theorem, when compared to Theorem 1.2(1), more clearly shows the relationship between $E$-reflexivity and $E$-hyperreflexivity. The theorem, when stated in terms of the seminorms $d_{Y}(, \mathscr{S})$ and $d_{E}(, \mathscr{S})$, is true for general seminorms. The proof is a simple application of the bipolar version of the Hahn-Banach theorem [RoRo].

Theorem 2.1. Suppose that $\mathscr{S}$ is a $\sigma(X, Y)$-closed linear subspace of $X$, and $K$ is a nonnegative number. The following are equivalent.

(1) $\mathscr{S}$ is E-hyperreflexive with hyperreflexivity constant at most $K$.

(2) ball $\left(\mathscr{S}^{\perp}\right) \subset K \overline{\mathrm{co}}\left(\widetilde{E} \cap \mathscr{S}^{\perp}\right)$.

Under the additional assumption that $Y$ is a Banach space and $X$ is the normed dual of $Y$, we obtain an analogue of the characterization of hyperreflexivity due to W. Arveson [Ar2]. Much of the proof is based on Arveson's ideas; however, Arveson's proof uses Hilbert-space concepts like the spectral theorem.

Theorem 2.2. Suppose that $(X, Y, E)$ is a normed reflexivity triple, $Y$ is a Banach space, and $X$ is the dual space $Y$. The following are equivalent.

(1) $\mathscr{S}$ is E-hyperreflexive.

(2) For each $f$ in $\mathscr{S}^{\perp}$ there is a sequence $\left\{e_{n}\right\}$ in $E \cap \mathscr{S}^{\perp}$ such that $\sum_{n=1}^{\infty}\left\|e_{n}\right\|<\infty$ and $f=\sum_{n=1}^{\infty} e_{n}$.

Proof. (1) $\Rightarrow(2)$. Suppose $\mathscr{S}$ is $E$-hyperreflexive. Let $\mathscr{B}=\operatorname{co}\left(\widetilde{E} \cap \mathscr{S}^{\perp}\right)$. Since $X$ is the dual of $Y$ and $\mathscr{B}$ is convex, it follows that the $\sigma(Y, X)$-closure of $\mathscr{B}$ coincides with the norm closure of $\mathscr{B}$. It follows from Theorem 2.1 that there is a positive number $K$ such that the norm closure of $K \mathscr{B}$ contains the unit ball of $\mathscr{S}^{\perp}$. Suppose that $f \in \mathscr{S}^{\perp}$. We can assume that $\|f\|=1$. Then there is an $f_{1}$ in $\mathscr{B}$ such that $\left\|f-K f_{1}\right\|<1 / 2$. Similarly, there is an $f_{2}$ in $\mathscr{B}$ such that $\left\|f-K f_{1}-(1 / 2) K f_{2}\right\|<1 / 4$. Proceeding inductively, we obtain a sequence $\left\{f_{n}\right\}$ in $\mathscr{B}$ such that $f=K \sum_{n=1}^{\infty} f_{n} / 2^{n-1}$. Since each $f_{n}$ is in $\mathscr{B}$, there are nonnegative numbers $t_{n 1}, \ldots, t_{n k_{n}}$ and vectors $e_{n 1}, \ldots, e_{n k_{n}}$ in $\widetilde{E} \cap \mathscr{S}^{\perp}$ such that

$$
\sum_{j=1}^{k_{n}} t_{n j}=1 \text { and } f_{n}=\sum_{j=1}^{k_{n}} t_{n j} e_{n j} \quad \text { for } n=1,2, \ldots .
$$

If we let $h_{n j}=K t_{n j} e_{n j} / 2^{n-1}$, then $\sum_{j, n}\left\|h_{n j}\right\|=K<\infty$, and $f=\sum_{j, n} h_{n j}$.

$(2) \Rightarrow(1)$. If $\left\{e_{n}\right\}$ is a sequence in $E \cap \mathscr{S}^{\perp}$ such that $s=\sum_{n}\left\|e_{n}\right\|<\infty$, then $\frac{1}{s} \sum_{n} e_{n}$ is in the closure $\mathscr{B}-$ of $\mathscr{B}=\operatorname{co}\left(\widetilde{E} \cap \mathscr{S}^{\perp}\right)$. It follows from (2) above that $\mathscr{S}^{\perp}=\bigcup\left\{n \mathscr{B}^{-}: n=1,2, \ldots\right\}$. It follows from the Baire category theorem that there is an $N$ such that ball $\left(\mathscr{S}^{\perp}\right) \subset N \overline{\mathrm{co}}\left(\widetilde{E} \cap \mathscr{S}^{\perp}\right)$. Hence, by Theorem 2.1 , we see that $\mathscr{S}$ is hyperreflexive.

Remark. It is easy to see from the proof of the preceding theorem that if condition (2) holds and if we define $\rho(f)=\inf \left\{\sum_{n}\left\|e_{n}\right\|: f=\sum_{n} e_{n}\right.$ and 
$\left.\left\{e_{n}\right\} \subset E \cap \mathscr{S}^{\perp}\right\}$ for each $f$ in $\mathscr{S}^{\perp}$, then $K_{E}(\mathscr{S})=\sup \left\{\rho(f): f \in \mathscr{S}^{\perp}\right.$, $\|f\|=1\}$.

We say that an $E$-hyperreflexive linear subspace $\mathscr{S}$ of $X$ is hereditarily $E$ hyperreflexive with respect to $Y$ if every $\sigma(X, Y)$-closed linear subspace of $\mathscr{S}$ is hyperreflexive. We say that $Y$ is E-elementary of order $r \geq 1$ on $\mathscr{S}$ if, for each $\varepsilon>0$ and each $f$ in $Y$, there is a $g$ in $E$ such that $f-g \in \mathscr{S}^{\perp}$ and such that $\|g\| \leq(r+\varepsilon)\|f \mid \mathscr{S}\|$. The following results generalize those in [KL].

Theorem 2.3. Suppose $\mathscr{S}$ is an E-hyperreflexive subspace of $X$. Then

(1) if $Y$ is complete, then $\mathscr{S}$ is hereditarily E-hyperreflexive with respect to $Y$ if and only if $Y$ is E-elementary on $\mathscr{S}$,

(2) if $Y$ is E-elementary of order $r$ on $\mathscr{S}$, then, for each $\sigma(X, Y)$-closed linear subspace $\mathscr{M}$ of $\mathscr{S}$, we have $K_{E}(\mathscr{M}) \leq(1+r)\left(1+K_{E}(\mathscr{S})\right)-1$.

Proof. (1). If $\mathscr{S}$ is hereditarily $E$-hyperreflexive, then $\mathscr{S}$ is hereditarily $E$ reflexive, which, by Theorem 1.2(3), implies that $\mathscr{S}$ is $E$-elementary. Conversely, suppose that $Y$ is $E$-elementary on $\mathscr{S}$ on $\mathscr{M}$ is a $\sigma(X, Y)$-closed linear subspace of $\mathscr{S}$. Suppose that $f \in \mathscr{M}^{\perp}$. Then there is a $g \in E \cap \mathscr{S}^{\perp}$ such that $f-g \in \mathscr{S}^{\perp} \subset \mathscr{M}^{\perp}$. Also, $f-g \in\|f-g\| K_{E}(\mathscr{S}) \overline{\operatorname{co}}\left(\widetilde{E} \cap \mathscr{S}^{\perp}\right) \subset$ $\|f-g\| K_{E}(\mathscr{S}) \overline{\operatorname{co}}\left(\widetilde{E} \cap \mathscr{M}^{\perp}\right)$. Thus

$$
f=(f-g)+g \in\left(\|f-g\| K_{E}(\mathscr{S})+\|g\|\right) \overline{\operatorname{co}}\left(\widetilde{E} \cap \mathscr{M}^{\perp}\right) .
$$

It follows that $\mathscr{M}^{\perp}$ is the union of $N \overline{\mathrm{co}}\left(\widetilde{E} \cap \mathscr{M}^{\perp}\right), N=1,2,3 \ldots$ Hence, by the Baire category theorem and Theorem $2.1, \mathscr{M}$ is $E$-hyperreflexive.

(2) If $\mathscr{S}$ is $E$-elementary of order $r,\|f\|=1$, and $\varepsilon>0$, then we could have chosen $g$ in the proof of (1) so that $\|g\| \leq r+\varepsilon$. In this case it would follow that $\|f-g\| \leq 1+r+\varepsilon$. Thus, since $\varepsilon>0$ was arbitrary, ball $\mathscr{M}^{\perp} \subset$ $\left[(1+r) K_{E}(\mathscr{S})+r\right] \overline{\operatorname{co}}\left(\widetilde{E} \cap \mathscr{M}^{\perp}\right)$, which implies $K_{E}(\mathscr{M}) \leq(1+r) K_{E}(\mathscr{S})+r=$ $(1+r)\left(1+K_{E}(\mathscr{S})\right)-\mathbf{i}$.

The following is a generalization of a theorem of R. Olin and J. Thomson [OT].

Theorem 2.4. Suppose $(X, Y, E)$ is a normed reflexivity triple, and $Y$ is the norm closure of $\mathrm{sp} E$. Suppose that $\mathscr{S}$ is a $\sigma(X, Y)$-closed linear subspace of $X$ and $Y$ is E-elementary of order $r$ on $\mathscr{S}$. Then $\mathscr{S}$ is $\sigma(X, \operatorname{sp} E)$-closed and the $\sigma(X, Y)$ and $\sigma(X, \operatorname{sp} E)$ topologies coincide on $\mathscr{S}$.

Proof. The fact that the $\sigma(X, Y)$ and the $\sigma(X, \operatorname{sp} E)$ topologies agree on $\mathscr{S}$ follows from $Y$ being $E$-elementary of $\mathscr{S}$. To show $\mathscr{S}$ is $\sigma(X, \mathrm{sp} E)$-closed, assume via contradiction that $x \notin \mathscr{S}$ and $x$ is in the $\sigma(X, \operatorname{sp} E)$-closure of $\mathscr{S}$. Then there is an $f$ in $\mathscr{S}^{\perp}$ such that $f(x)=1$. Since $Y$ is the norm closure of $\operatorname{sp} E$, there is a sequence $\left\{g_{n}\right\}$ in $\operatorname{sp} E$ such that $\sum_{n=1}^{\infty}\left\|g_{n}\right\|<\infty$ and $f=\sum_{n=1}^{\infty} g_{n}$. Since $Y$ is $E$-elementary of order $r$ on $\mathscr{S}$, there are sequences $\left\{e_{n}\right\}$ and $\left\{h_{n}\right\}$ in $E$ such that, for each positive integer $n$, we have $g_{n}-e_{n}, h_{n}-\sum_{j>n} g_{j} \in \mathscr{S}^{\perp}$ and $\left\|e_{n}\right\| \leq(r+1)\left\|g_{n}\right\|,\left\|h_{n}\right\| \leq(r+1) \sum_{j>n}\left\|g_{j}\right\|$. Hence $\left\|h_{n}\right\| \rightarrow 0$, which implies $h_{n}(x) \rightarrow 0$. Since $x$ is in the $\sigma(X, \operatorname{sp} E)$ closure of $\mathscr{S}$ and since $g_{n}-e_{n} \in \mathscr{S}^{\perp} \cap \operatorname{sp} E$, we have $e_{n}(x)=g_{n}(x)$ for each $n$. Similarly, $e_{1}(x)+\cdots+e_{n}(x)=-h_{n}(x)$ for each $n$. The desired 
contradiction is

$$
1=f(x)=\sum_{n=1}^{\infty} g_{n}(x)=\sum_{n=1}^{\infty} e_{n}(x)=-\lim h_{n}(x)=0 .
$$

In certain cases we can extend the result on reflexive graphs (Theorem 1.3) to hyperreflexive graphs. In the Hilbert space case this yields new results, including the result that if $S$ is the unilateral shift operator and $T$ is a polynomially bounded operator, then $S \oplus T$ is hyperreflexive (see Theorems 6.9 and 6.14).

Theorem 2.5. Suppose that $\left(X_{i}, Y_{i}, E_{i}\right)$ is a normed reflexivity triple for $i=$ $1,2, \mathscr{S}$ is an $E_{1}$-hyperreflexive linear subspace of $X_{1}, s>0, r>0$, and $\rho: \mathscr{S} \rightarrow X_{2}$ is a linear mapping such that

(1) every functional in $Y_{2}$ of norm 1 can be written as an absolutely convergent series from $E_{2}$ whose sum of norms is less than $s$,

(2) $\mathscr{S}$ is $E_{1}$-elementary of order $r$.

(3) $\rho$ is $\sigma\left(X_{1}, Y_{1}\right)-\sigma\left(X_{2}, Y_{2}\right)$ continuous. Then

(4) $\operatorname{graph}(\rho)$ is an $E_{1} \times E_{2}$-hyperreflexive linear subspace of $X_{1} \times X_{2}$, and

(5) $Y_{1} \times Y_{2}$ is $E_{1} \times E_{2}$-elementary on $\operatorname{graph}(\rho)$.

Proof. Without loss of generality, we can give $X_{1} \times X_{2}$ the norm $\left\|\left(x_{1}, x_{2}\right)\right\|=$ $\max \left(\left\|x_{1}\right\|,\left\|x_{2}\right\|\right)$. Suppose that $\varepsilon>0$ and $\varphi=\left(\varphi_{1}, \varphi_{2}\right) \in Y_{1} \times Y_{2}$ and $\varphi \in$ $\operatorname{graph}(\rho)^{\perp}$ and $\|\varphi\|=\left\|\varphi_{1}\right\|+\left\|\varphi_{2}\right\|=1$. By hypothesis, there is a sequence $\left\{f_{n}\right\}$ in $E_{2}$ such that $\varphi_{2}=\sum_{n} f_{n}$ and $\sum_{n}\left\|f_{n}\right\| \leq s\left\|\varphi_{2}\right\|$. Since $\mathscr{S}$ is $E_{1}$-elementary of order $r$, there is a sequence $\left\{e_{n}\right\}$ in $E_{1}$ such that, for each $n, e_{n} \mid \mathscr{S}=f_{n} \circ \rho$ and $\left\|e_{n}\right\| \leq(r+\varepsilon)\|\rho\|\left\|f_{n}\right\|$. Hence, each $\left(-e_{n}, f_{n}\right)$ is in $\operatorname{graph}(\rho)^{\perp} \cap\left(E_{1} \times E_{2}\right)$, and $\sum_{n}\left\|\left(-e_{n}, f_{n}\right)\right\|=\sum_{n}\left\|e_{n}\right\|+\left\|f_{n}\right\| \leq[(r+\varepsilon)\|\rho\|+1] s\left\|\varphi_{2}\right\|$. Also, $\varphi=$ $\left(\varphi_{1}+\sum_{n} e_{n}, 0\right)+\sum_{n}\left(-e_{n}, f_{n}\right)$; therefore, $e=\varphi_{1}+\sum_{n} e_{n} \in \mathscr{S}^{\perp}$ and has norm at most $\left\|\varphi_{1}\right\|+(r+\varepsilon) s\|\rho\|\left\|\varphi_{2}\right\|$.

Since $\mathscr{S}$ is $E_{1}$-hyperreflexive,

$$
e \in K_{E_{1}}(\mathscr{S}) \overline{\operatorname{co}}\left(\widetilde{E}_{1} \cap \mathscr{S}^{\perp}\right)\left[\left\|\varphi_{1}\right\|+(r+\varepsilon) s\|\rho\|\left\|\varphi_{2}\right\|\right] .
$$

Since $\overline{\operatorname{co}}\left(\widetilde{E}_{1} \cap \mathscr{S}^{\perp}\right) \times\{0\} \subset \overline{\operatorname{co}}\left(\operatorname{graph}(\rho)^{\perp} \cap\left(\widetilde{E}_{1} \times E_{2}\right)\right)$, thus $\varphi$ is in

$$
K \overline{\operatorname{co}}\left(\operatorname{graph}(\rho)^{\perp} \cap\left(\widetilde{E}_{1} \times E_{2}\right)\right)
$$

where $K=\left[K_{E_{1}}(\mathscr{S})\left[\left\|\varphi_{1}\right\|+(r+\varepsilon) s\|\rho\|\left\|\varphi_{2}\right\|\right]+[(r+\varepsilon)\|\rho\|+1] s\left\|\varphi_{2}\right\|\right]$. Hence, by Theorem 2.1, $\operatorname{graph}(\rho)$ is $E_{1} \times E_{2}$-hyperreflexive.

Remarks. 1. If in the preceding theorem we give $X_{1} \times X_{2}$ the norm

$$
\left\|\left(x_{1}, x_{2}\right)\right\|=\max \left(\left\|x_{1}\right\|,\left\|x_{2}\right\|\right),
$$

then the preceding proof shows that the constant of hyperreflexivity of $\operatorname{graph}(\rho)$ does not exceed $\max \left(K_{E_{1}}(\mathscr{S}),\left[K_{E_{1}}(\mathscr{S}) r s\|\rho\|+[r\|\rho\|+1] s\right]\right)$. Also $Y_{1} \times Y_{2}$ is $E_{1} \times E_{2}$-elementary of order $r \cdot \max (1,\|\rho\|)$ on $\operatorname{graph}(\rho)$.

2. Suppose $X_{2}=Y_{2}=l^{2}$ with the natural duality and suppose $E$ is the set of all scalar multiples of the vectors $e_{n}=(1,0, \ldots, 1 / n, 0, \ldots)$ with the $1 / n$ appearing in the $n$th coordinate. It is clear that $\overline{c o}(\widetilde{E})$ has empty interior. Thus $\{0\}$ is not an $E_{2}$-hyperreflexive subspace of $X_{2}$. Suppose $\left(X_{1}, Y_{1}, E_{1}\right)$ is a normed reflexivity triple such that $\mathscr{S}=\{0\}$ is an $E_{1}$-hyperreflexive subspace of $X_{1}$, and define $\rho: \mathscr{S} \rightarrow X_{2}$ by $\rho(0)=0$. Then $\operatorname{graph}(\rho)$ is not 
$E_{1} \times E_{2}$-hyperreflexive. Hence the hypothesis that every element of $Y_{2}$ is an absolutely convergent sum of a sequence in $E_{2}$ cannot be removed. It would be interesting to know if it could be replaced by the condition that $\{0\}$ is $E_{2}$-hyperreflexive.

We next consider the notions of relative reflexivity and hyperreflexivity. These notions are the key links between our general results and some of their applications. Suppose that $(X, Y, E)$ is a reflexivity triple and $\mathscr{M}$ is a subspace of $X$. A subspace $\mathscr{S}$ of $\mathscr{M}$ is E-reflexive relative to $\mathscr{M}$ if $\operatorname{Ref}_{E}(\mathscr{S}) \cap \mathscr{M}=$ $\mathscr{S}$. Note that $\left(\mathscr{M}, Y / \mathscr{M}^{\perp}, E / \mathscr{M}^{\perp}\right)$ is a reflexivity triple, and $\mathscr{S}$ is $E$ reflexive with respect to $\mathscr{M}$ if and only if $\mathscr{S}$ is $E / \mathscr{M}^{\perp}$-reflexive.

Next suppose that $(X, Y, E)$ is a normed reflexivity triple and $\mathscr{M}$ is a subspace of $X$. A subspace $\mathscr{S}$ of $\mathscr{M}$ is $E$-hyperreflexive relative to $\mathscr{M}$ if there is a smallest number $K=K_{E}(\mathscr{S}, \mathscr{M})$ such that $d_{Y}(x, \mathscr{S}) \leq K d_{E}(x, \mathscr{S})$ for every $x$ in $\mathscr{M}$.

The key result concerning relative reflexivity and relative hyperreflexivity is the transitivity of the relations.

Theorem 2.6. (1) If $\mathscr{S}$ is E-reflexive relative to $\mathscr{M}$ and $\mathscr{M}$ is E-reflexive relative to $\mathscr{N}$, then $\mathscr{S}$ is E-reflexive relative to $\mathscr{N}$.

(2) If $\mathscr{S}$ is E-hyperreflexive relative to $\mathscr{M}$ and $\mathscr{M}$ is E-hyperreflexive relative to $\mathscr{N}$, and if $\operatorname{dist}(x, \mathscr{M})=d_{Y}(x, \mathscr{M})$ for every $x$ in $\mathscr{N}$, then $\mathscr{S}$ is E-hyperreflexive relative to $\mathscr{N}$, and

$$
K_{E}(\mathscr{S}, \mathscr{N}) \leq\left[1+K_{E}(\mathscr{S}, \mathscr{M})\right]\left[1+K_{E}(\mathscr{M}, \mathscr{N})\right]=1 .
$$

Proof. (1) Since $\mathscr{S}=\operatorname{Ref}_{E}(\mathscr{S}) \cap \mathscr{M}$ and $\mathscr{M}=\operatorname{Ref}_{E}(\mathscr{M}) \cap \mathscr{N}$, we have $\mathscr{S} \subset$ $\operatorname{Ref}_{E}(\mathscr{S}) \cap \mathscr{N} \subset \operatorname{Ref}_{E}(\mathscr{S}) \cap \operatorname{Ref}_{E}(\mathscr{M}) \cap \mathscr{N}=\operatorname{Ref}_{E}(\mathscr{S}) \cap \mathscr{M}=\mathscr{S}$.

(2) Suppose $\varepsilon>0$ and $x \in \mathscr{N}$. Since

$$
\operatorname{dist}(x, \mathscr{M})=d_{Y}(x, \mathscr{M}) \leq K_{E}(\mathscr{M}, \mathscr{N}) d_{E}(x, \mathscr{M}),
$$

there is a $z$ in $\mathscr{M}$ such that

$$
\|x-z\| \leq K_{E}(\mathscr{M}, \mathscr{N}) d_{E}(x, \mathscr{M})+\varepsilon \leq K_{E}(\mathscr{M}, \mathscr{N}) d_{E}(x, \mathscr{S})+\varepsilon .
$$

However,

$$
\begin{aligned}
d_{Y}(x, \mathscr{S}) \leq d_{Y}(x-z, \mathscr{S})+d_{Y}(z, \mathscr{S}) \leq\|x-z\|+K_{E}(\mathscr{S}, \mathscr{M}) d_{E}(z, \mathscr{S}) \\
\leq K_{E}(\mathscr{M}, \mathscr{N}) d_{E}(x, \mathscr{S})+\varepsilon+K_{E}(\mathscr{S}, \mathscr{M})\left[d_{E}(z-x, \mathscr{S})+d_{E}(x, \mathscr{S})\right] \\
\leq K_{E}(\mathscr{M}, \mathscr{N}) d_{E}(x, \mathscr{S})+\varepsilon+K_{E}(\mathscr{S}, \mathscr{M})\left[\|x-z\|+d_{E}(x, \mathscr{S})\right] \\
\leq K_{E}(\mathscr{M}, \mathscr{N}) d_{E}(x, \mathscr{S})+\varepsilon \\
\quad+K_{E}(\mathscr{S}, \mathscr{M})\left[K_{E}(\mathscr{M}, \mathscr{N}) d_{E}(x, \mathscr{S})+\varepsilon+d_{E}(x, \mathscr{S})\right] .
\end{aligned}
$$

But $\varepsilon>0$ was arbitrary; thus

$$
d_{Y}(x, \mathscr{S}) \leq\left[\left[1+K_{E}(\mathscr{S}, \mathscr{M})\right]\left[1+K_{E}(\mathscr{M}, \mathscr{N})\right]-1\right] d_{E}(x, \mathscr{S})
$$

for every $x$ in $\mathscr{N}$.

Remark. In part (2) of Theorem 2.6, if the condition $\operatorname{dist}(x, \mathscr{M})=d_{Y}(x, \mathscr{M})$ is replaced by $\operatorname{dist}(x, \mathscr{M}) \leq s d_{Y}(x, \mathscr{M})$ for some positive number $s$, we can still conclude that $\mathscr{S}$ is $E$-hyperreflexive relative to $\mathscr{N}$.

We conclude this section with two results on the intersection of hyperreflexive subspaces. It is clear that a subspace is $E$-reflexive if and only if it is the 
intersection of the kernels of a collection of functions in $E$. It follows that the intersection of an arbitrary nonempty family of $E$-reflexive subspaces is $E$-reflexive. On the other hand, if $e \in E$ and $\|e\|=1$, and $M=\operatorname{ker}(e)$, $M$ is $E$-hyperreflexive with $K_{E}(M)=1$. Hence every $E$-reflexive subspace is an intersection of $E$-hyperreflexive reflexive subspaces with hyperreflexivity constant 1 . Since not every $E$-reflexive subspace is $E$-hyperreflexive, it follows that $E$-hyperreflexivity is not preserved under intersections. However, under certain circumstances, something useful can be said about intersections and hyperreflexivity.

The first result concerns intersections of downwardly directed collections, which, in some cases, reduces the problem of arbitrary intersections to finite intersections.

Theorem 2.7. Suppose that $(X, Y, E)$ is a normed reflexivity triple and ball $(X)$ is $\sigma(X, Y)$-compact. If $\left\{\mathscr{S}_{\lambda}: \lambda \in \Lambda\right\}$ is a downwardly directed family of $\sigma(X, Y)$ closed linear subspaces of $X$, and $\mathscr{S}=\bigcap\left\{\mathscr{S}_{\lambda}: \lambda \in \Lambda\right\}$, then

(1) $d_{Y}(x, \mathscr{M})=\operatorname{dist}(x, \mathscr{M})$ for every $\sigma(X, Y)$-closed linear subspace $\mathscr{M}$ of $X$ and every $x$ in $X$,

(2) $d_{Y}(x, \mathscr{S})=\sup _{\lambda} d_{Y}\left(x, \mathscr{S}_{\lambda}\right)$ for every $x$ in $X$,

(3) $K_{E}(\mathscr{S}) \leq \sup _{\lambda} K_{E}\left(\mathscr{S}_{\lambda}\right)$.

Proof. (1) This follows from [HN1, Lemma 2.4].

(2) Suppose $x \in X$. It is clear that $d_{Y}(x, \mathscr{S}) \geq \sup _{\lambda} d_{Y}\left(x, \mathscr{S}_{\lambda}\right)$. For the other direction suppose $\varepsilon>0$, and let $B$ be the closed ball centered at $x$ with radius $r=\varepsilon+\sup _{\lambda} d_{Y}\left(x, \mathscr{S}_{\lambda}\right)$. Since $B$ is homeomorphic to ball $X$, we know that $B$ is $\sigma(X, Y)$-compact. Thus, by (1), $\operatorname{dist}\left(x, \mathscr{S}_{\lambda}\right)<r$ for every $\lambda$ in $\Lambda$ implies that the set $\left\{B \cap \mathscr{S}_{\lambda}: \lambda \in \Lambda\right\}$ is a downwardly directed collection of nonempty $\sigma(X, Y)$-compact sets. Hence $B \cap \mathscr{S} \neq \varnothing$. This implies $d_{Y}(x, \mathscr{S}) \leq \sup _{\lambda} d_{Y}\left(x, \mathscr{S}_{\lambda}\right)+\varepsilon$. Since $\varepsilon$ was arbitrary, it follows that $d_{Y}(x, \mathscr{S}) \leq \sup _{\lambda} d_{Y}\left(x, \mathscr{S}_{\lambda}\right)$.

(3) This follows immediately from (2).

Another result on intersections is the following intersection theorem.

Theorem 2.8. Suppose that $(X, Y, E)$ is a normed reflexivity triple, $X$ is a Banach space, and $\mathscr{M}$ and $\mathscr{N}$ are E-hyperreflexive subspaces of $X$ such that

(1) $d_{Y}(x, \mathscr{M})=\operatorname{dist}(x, \mathscr{M})$ for every $x \in X$, and

(2) $\mathscr{M}+\mathscr{N}$ is norm closed.

Then $\mathscr{M} \cap \mathscr{N}$ is E-hyperreflexive.

Proof. Let $\rho: \mathscr{M} \rightarrow X / \mathscr{N}$ be the quotient map restricted to $\mathscr{M}$. Since $\mathscr{M}+\mathscr{N}$ is closed, we have that $\rho(\mathscr{M})$ is closed. Since $\operatorname{ker}(\rho)=\mathscr{M} \cap \mathscr{N}$, it follows that $\mathscr{M} /(\mathscr{M} \cap \mathscr{N})$ is isomorphic to $\rho(\mathscr{M})$. Hence there is a positive number $\xi$ such that, for every $x$ in $\mathscr{M}$, we have

$\operatorname{dist}(x, \mathscr{M} \cap \mathscr{N}) \leq \xi \operatorname{dist}(x, \mathscr{N}) \leq \xi K_{E}(\mathscr{N}) d_{Y}(x, \mathscr{N}) \leq \xi K_{E}(\mathscr{N}) d_{Y}(x, \mathscr{M} \cap \mathscr{N})$. Hence $\mathscr{M} \cap \mathscr{N}$ is relatively $E$-hyperreflexive in $\mathscr{M}$. It follows from part (2) of Theorem 2.6 that $\mathscr{M} \cap \mathscr{N}$ is $E$-hyperreflexive.

\section{DiRECT INTEGRALS}

In this section we develop analogues in our general setting of direct integrals, and we prove general versions of reflexivity results concerning direct integrals 
[AFG, HN1, HN2]. We also prove a new result in our general setting that translates in the Hilbert space case to the theorem that a direct integral of algebras is hyperreflexive if and only if almost every integrand is hyperreflexive and the constants of hyperreflexivity are essentially bounded.

We shall need some results concerning measurable cross-sections. Recall that an analytic space is a metrizable topological space that is a continuous image of a complete separable metric space. Throughout this section $(\Omega, \mathscr{M}, \mu)$ is a complete $\sigma$-finite measure space. Suppose $A$ is an analytic space. Give the product $\Omega \times A$ the product Borel structure, and let $\pi_{1}: \Omega \times A \rightarrow \Omega$ be the projection map. If $Q \subset \Omega \times A, \omega \in \Omega$, let $Q_{\omega}=\{a \in A:(\omega, a) \in$ $Q$ \}. A subset of a topological space is absolutely measurable if it is measurable with respect to every $\sigma$-finite Borel measure. Similarly, a function is absolutely measurable if it is measurable with respect to every $\sigma$-finite Borel measure. Finally, a cross-section for a function $f: A \rightarrow B$ is a function $\alpha: f(A) \rightarrow A$ such that $f(\alpha(b))=b$ for every $b \in f(A)$. We will make use of the following results, which can be found in [Hi, S-B, Ar3].

Proposition 3.1. Suppose $A$ is an analytic space.

(1) If $Q$ is a measurable subset of $\Omega \times A$, then $\pi_{1}(Q) \in \mathscr{M}$, and there is a measurable function from $\pi_{1}(Q)$ to $A$ whose graph is contained in $Q$.

(2) If $B$ is a separable metric space, $f: A \rightarrow B$ is measurable, then $f(A)$ is an absolutely measurable subset of $B$, and $f$ has an absolutely measurable cross-section.

(3) Suppose $Q_{\omega}$ is closed for each $\omega$ in $\Omega$. Then $Q$ is measurable if and only if there is a sequence $\left\{\varphi_{n}\right\}$ of measurable functions from $\pi_{1}(Q)$ to $A$ such that $\left\{\varphi_{n}(\omega): n \geq 1\right\}$ is dense in $Q_{\omega}$ for every $\omega$ in $\Omega$.

Throughout this section, we assume that $(X, Y, E)$ is a normed reflexivity triple such that

(Ai) the unit ball of $X$ is $\sigma(X, Y)$-metrizable, separable and analytic,

(Aii) the unit ball of $Y$ is $\sigma(Y, X)$-metrizable, separable and analytic,

(Aiii) $\{e \in E:\|e\| \leq 1\}$ is $\sigma(Y, X)$-analytic,

(Aiv) the map $\langle\rangle:, X \times Y \rightarrow F$ defined by $\langle x, f\rangle=f(x)$ is measurable with respect to the product Borel structure on $X \times Y$ given by the $\sigma(X, Y)$ and $\sigma(Y, X)$ topologies.

Remarks. 1. It follows from (Ai) above that the map $x \mapsto\|x\|$ is absolutely $\sigma(X, Y)$-measurable on $X$ and it follows from (Aii) that the map $y \mapsto\|y\|$ is absolutely $\sigma(Y, X)$-measurable on $Y$. If $\operatorname{ball}(X)$ is a Borel set, then the map $x \mapsto\|x\|$ is Borel measurable.

2. If $X$ is norm separable, then the norm Borel subsets of $X$ are absolutely measurable with respect to the $\sigma(X, Y)$-Borel sets. To see this, first note that the preceding remark implies that open balls in $X$ are absolutely $\sigma(X, Y)$ measurable, and the norm separability of $X$ implies that every norm-open subset of $X$ is a countable union of open balls. Thus every norm open subset of $X$ is absolutely $\sigma(X, Y)$-measurable. If $\operatorname{ball}(X)$ is a $\sigma(X, Y)$-Borel set, then the norm-Borel sets coincide with the $\sigma(X, Y)$-Borel sets. Clearly, this remark remains true if $X$ and $Y$ are interchanged.

3. If either $X$ is norm separable or $\operatorname{ball}(X)$ is a $\sigma(X, Y)$-Borel set, then (Aiv) above is implied by (Ai), (Aii), and (Aiii). To see this, note that it is 
enough to show that $\langle$,$\rangle restricted to \operatorname{ball}(X) \times$ ball $(Y)$ is measurable. However, if $X$ is norm separable or $\operatorname{ball}(X)$ is a $\sigma(X, Y)$-Borel set, the preceding remark shows that it is enough to show that the restriction of $\langle$,$\rangle to$ $\operatorname{ball}(X) \times \operatorname{ball}(Y)$ is measurable with respect to the norm topology on ball $(X)$ and the $\sigma(Y, X)$-topology on ball $(Y)$. However, $\langle$,$\rangle is continuous with re-$ spect to these topologies. To see this, note that if $\left\{x_{n}\right\}$ is a sequence in ball $(X)$ that is norm convergent to $x$ and if $\left\{f_{n}\right\}$ is a sequence in $\operatorname{ball}(Y)$ that is $\sigma(Y, X)$-convergent to $f$, then we have $\left|\left\langle x_{n}, f_{n}\right\rangle-\langle x, f\rangle\right| \leq\left\|x_{n}-x\right\|\left\|f_{n}\right\|+$ $\left|\left\langle x, f_{n}\right\rangle-\langle x, f\rangle\right| \rightarrow 0$ as $n \rightarrow \infty$. The validity of this remark is also invariant under the interchange of $X$ and $Y$.

Throughout this section we assume that $1 \leq p, q \leq \infty$ and $1 / p+1 / q=$ 1. We define $L_{Y}^{p}(\mu, X)$ and $L_{X}^{q}(\mu, Y)$ by $L_{Y}^{p}(\mu, X)=\{f \mid f: \Omega \rightarrow X, f$ is $\sigma(X, Y)$-measurable, $\|f(\omega)\|$ is in $\left.L^{p}(\mu)\right\}$, and, similarly, $L_{X}^{q}(\mu, Y)=$ $\left\{g \mid g: \Omega \rightarrow Y, g\right.$ is $\sigma(Y, X)$-measurable, $\|g(\omega)\|$ is in $\left.L^{q}(\mu)\right\}$. We also define $L_{X}^{q}(\mu, E)=\left\{g \in L_{X}^{q}(\mu, Y): g(\omega) \in E\right.$ a.e. $\left.(\mu)\right\}$. We define a duality $\langle$,$\rangle between L_{Y}^{p}(\mu, X)$ and $L_{X}^{q}(\mu, Y)$ by

$$
\langle f, g\rangle=\int_{\Omega}\langle f(\omega), g(\omega)\rangle d \mu(\omega) .
$$

It follows from (Aiv) above that the integrand is measurable and from Hölder's inequality that it is in $L^{1}(\mu)$.

As usual, we identify two functions that agree almost everywhere. It is clear that the norms

$$
\|f\|_{p}=\left(\int_{\Omega}\|f(\omega)\|^{p} d \mu(\omega)\right)^{1 / p}
$$

and

$$
\|g\|_{q}=\left(\int_{\Omega}\|g(\omega)\|^{q} d \mu(\omega)\right)^{1 / q}
$$

make $L_{Y}^{p}(\mu, X)$ and $L_{X}^{q}(\mu, Y)$ normed linear spaces.

Definition. A family $\left\{\mathscr{S}_{\omega}: \omega \in \Omega\right\}$ of $\sigma(X, Y)$-closed linear subspaces of $X$ is attainable if there is a sequence $\left\{f_{n}\right\}$ of measurable functions from $\Omega$ to $X$ such that

$$
\mathscr{S}_{\omega}=\overline{\mathrm{sp}}\left\{f_{1}(\omega), f_{2}(\omega), \ldots\right\} \text { a.e. }(\mu) .
$$

The family $\left\{\mathscr{S}_{\omega}: \omega \in \Omega\right\}$ is measurable if there is a sequence $\left\{h_{n}\right\}$ of measurable functions from $\Omega$ into ball $(X)$ such that $\left\{h_{n}(\omega): n \geq 1\right\}$ is $\sigma(X, Y)$ dense in ball $\left(\mathscr{S}_{\omega}\right)$ a.e. $(\mu)$. Similar definitions are made for families of subspaces of $Y$.

Lemma 3.2. Suppose $\left\{\mathscr{S}_{\omega}: \omega \in \Omega\right\}$ is an attainable family of $\sigma(X, Y)$-closed linear subspaces of $X$. Then

(1) $\left\{\mathscr{S}_{\omega}: \omega \in \Omega\right\}$ is measurable,

(2) $\left\{\mathscr{S}_{\omega}^{\perp}: \omega \in \Omega\right\}$ is measurable, and

(3) there is a sequence $\left\{g_{n}\right\}$ of measurable functions from $\Omega$ into $\widetilde{E}$ such that $\left\{g_{n}(\omega): n \geq 1\right\}$ is $\sigma(Y, X)$-dense in $\mathscr{S}_{\omega}^{\perp} \cap \widetilde{E}$ a.e. $(\mu)$.

Proof. (2) Suppose $\left\{f_{n}\right\}$ is a sequence of measurable functions from $\Omega$ to $X$ such that $\left\{f_{n}(\omega): n \geq 1\right\}^{-}=\mathscr{S}_{\omega}$ a.e. $(\mu)$. By throwing away a set of measure zero, we can assume that $\left\{f_{n}(\omega): n \geq 1\right\}^{-}=\mathscr{S}_{\omega}$ for every $\omega$ in $\Omega$. For each 
positive integer $n$, define $\Gamma_{n}: \Omega \times$ ball $(Y) \rightarrow F$ by $\Gamma_{n}(\omega, g)=\left\langle f_{n}(\omega), g\right\rangle$. Since $\langle$,$\rangle is measurable on X \times Y$ and the map $(\omega, g) \mapsto\left(\varphi_{n}(\omega), g\right)$ is measurable, it follows that $\Gamma_{n}$ is measurable for $n \geq 1$. Therefore, $Q=$ $\bigcap_{n \geq 1} \Gamma_{n}^{-1}(\{0\})$ is measurable in $\Omega \times$ ball $(Y)$. Hence, by Proposition 3.1 , there is a sequence $\left\{h_{n}\right\}$ of measurable functions from $\Omega$ in to ball $(Y)$ such that, for every $\omega$ in $\Omega,\left\{h_{n}(\omega): n \geq 1\right\}^{-}=\left\{g \in \operatorname{ball}(Y):\left\langle f_{k}(\omega), g\right\rangle=0\right.$ for $k \geq 1\}=\operatorname{ball}\left(\mathscr{S}_{\omega}^{\perp}\right)$ a.e. $(\mu)$. Hence $\left\{\mathscr{S}_{\omega}^{\perp}: \omega \in \Omega\right\}$ is a measurable family.

(1) This follows from (2) by interchanging the roles of $\mathscr{S}_{\omega}^{\perp}$ and $\mathscr{S}_{\omega}=$ $\left(\mathscr{S}_{\omega}^{\perp}\right)_{\perp}$.

(3) This follows as in the proof of (2) with $\widetilde{E}$ replacing ball $(Y)$.

We now assume that $\mathscr{X}$ is a vector space of (equivalence classes of measurable functions from $\Omega$ to $X$ such that

(Bi) If $f \in \mathscr{X}, g: \Omega \rightarrow X$ is measurable, $\|g(\omega)\| \leq\|f(\omega)\|$ a.e. $(\mu)$, then $g \in \mathscr{X}$.

(Bii) If $M \in \mathscr{M}$ and $\mu(M)>0$, then there is an $A \subset M$ with $\mu(A)>0$ and an $f$ in $\mathscr{X}$ such that $f(\omega) \neq 0$ for all $\omega$ in $A$.

Similarly, we assume that $\mathscr{Y}$ is a vector space of measurable functions from $\Omega$ to $Y$ so that

(Biii) If $f \in \mathscr{Y}, g: \Omega \rightarrow Y$ measurable, $\|g(\omega)\| \leq\|f(\omega)\|$ a.e. $(\mu)$, then $g \in \mathscr{Y}$.

(Biv) If $M \in \mathscr{M}$ and $\mu(M)>0$, then there is an $A \subset M$ with $\mu(A)>0$ and a $g$ in $\mathscr{Y}$ such that $g(\omega) \neq 0$ for all $\omega$ in $A$.

We also assume

(Bv) If $f \in \mathscr{X}$ and $g \in \mathscr{Y}$, then the function $\langle f, g\rangle$ on $\Omega$ is defined by $\langle f, g\rangle(\omega)=\langle f(\omega), g(\omega)\rangle$ is in $L^{1}(\mu)$.

We define $\mathscr{E}=\{g \in \mathscr{Y}: g(\omega) \in E$ a.e. $(\mu)\}$.

Examples. 1. Let $\mathscr{X}=L_{Y}^{p}(\mu, X), \mathscr{Y}=L_{X}^{q}(\mu, Y), \mathscr{E}=L_{X}^{q}(\mu, E)$.

2. $\mathscr{X}=\left\{f \in L_{Y}^{1}(\mu, X): \mu(\{\omega: f(\omega) \neq 0\})<\infty\right\}, \mathscr{Y}=L_{X}^{3}(\mu, Y), \mathscr{E}=$ $L_{X}^{3}(\mu, E)$.

3. Suppose $\psi: \Omega \rightarrow(0, \infty)$ is a measurable function. Let $\mathscr{X}=\psi L_{Y}^{p}(\mu, X)$, and let $\mathscr{Y}=\frac{1}{\psi} L_{X}^{q}(\mu, Y)$.

Lemma 3.3. ( $\mathscr{X}, \mathscr{Y}, \mathscr{E})$ is a reflexivity triple.

Proof. We need only show that $\mathscr{E}_{\perp}=\{0\}$. Suppose $f \in \mathscr{X}$ and $f \neq 0$. It follows from (iv) above that there is a set $A$ in $\mathscr{M}$ with $\mu(A)>0$ and a $g$ in $\mathscr{Y}$ such that $f(\omega) \neq 0$ and $g(\omega) \neq 0$ on $A$. Consider the set $Q=\{(x, e) \in \operatorname{ball}(X) \times \widetilde{E}:\langle x, e\rangle>0\}=\widetilde{E} \cap\left[\langle,\rangle^{-1}(0, \infty)\right]$, which is analytic since it is a Borel subset of an analytic set. Thus $\pi_{1}: Q \rightarrow \operatorname{ball}(X)$ has an absolutely measurable cross-section $\alpha$. Hence $\beta=\pi_{2} \circ \alpha: \pi_{1}(Q) \rightarrow$ $\widetilde{E}$ is absolutely measurable. Define $h(\omega)=\|g(\omega)\| \beta(f(\omega) /\|f(\omega)\|)$ for $\omega$ in $A$ and $h(\omega)=0$ off $A$. Since $h$ is measurable and $\|h(\omega)\| \leq\|g(\omega)\|$ a.e. $(\mu)$, it follows that $h \in \mathscr{Y}$. Since $h(\omega) \in E$ a.e. $(\mu)$, it follows that $h \in \mathscr{E}$. Clearly, $\langle f(\omega), h(\omega)\rangle>0$ on $A$, and since $\mu(A)>0$ and $\langle f, h\rangle=$ $\int_{A}\langle f(\omega), h(\omega)\rangle d \mu(\omega)$, we conclude that $\langle f, h\rangle>0$.

Hence $\mathscr{E}_{\perp}=\{0\}$.

If $\left\{\mathscr{S}_{\omega}: \omega \in \Omega\right\}$ is a family of subsets of $X$, we define the direct inte- 
gral of the $\mathscr{S}_{\omega}$ 's with respect to $\mathscr{X}$, denoted $\int_{\Omega, \mathscr{X}}^{\oplus} \mathscr{S}_{\omega} d \mu(\omega)$, to be the set of all $f$ in $\mathscr{Z}$ such that $f(\omega) \in \mathscr{S}_{\omega}$ a.e. $(\mu)$. For such an $f$ we write $f=\int_{\Omega, \mathscr{X}}^{\oplus} f(\omega) d \mu(\omega)$. If $\mathscr{X}=L_{Y}^{p}(\mu, X)$, we write $\int_{\Omega, p}^{\oplus} \mathscr{S}_{\omega} d \mu(\omega)$ for $\int_{\Omega, \mathscr{L}}^{\oplus} \mathscr{S}_{\omega} d \mu(\omega)$.

Remark. Using the direct integral notation, we see that $\mathscr{X}=\int_{\Omega, \mathscr{Z}}^{\oplus} X d \mu(\omega)$, and $\mathscr{Y}=\int_{\Omega, \mathscr{Y}}^{\oplus} Y d \mu(\omega)$, and $\mathscr{E}=\int_{\Omega, \mathscr{Y}}^{\oplus} E d \mu(\omega)$.

Theorem 3.4. Suppose $\left\{\mathscr{S}_{\omega}: \omega \in \Omega\right\}$ and $\left\{\mathscr{T}_{\omega}: \omega \in \Omega\right\}$ are measurable families of $\sigma(X, Y)$-closed linear subspaces of $X$. Then

1. There is a sequence $\left\{f_{n}\right\}$ in $\mathscr{X}$ such that $\left\{f_{n}(\omega): n \geq 1\right\}^{-}=\mathscr{S}_{\omega}$ a.e. $(\mu)$,

2. $\int_{\Omega, \mathscr{L}}^{\oplus} \mathscr{S}_{\omega} d \mu(\omega)=\int_{\Omega, \mathscr{L}}^{\oplus} \mathscr{T}_{\omega} d \mu(\omega)$ if and only if $\mathscr{S}_{\omega}=\mathscr{T}_{\omega}$ a.e. $(\mu)$,

3. $\left(\int_{\Omega, \mathscr{Z}}^{\oplus} \mathscr{S}_{\omega} d \mu(\omega)\right)^{\perp}=\int_{\Omega, \mathscr{Y}}^{\oplus} \mathscr{S}_{\omega}^{\perp} d \mu(\omega)$,

4. $\left(\int_{\Omega, \mathscr{L}}^{\oplus} \mathscr{S}_{\omega} d \mu(\omega)\right)^{\perp} \cap \mathscr{E}=\int_{\Omega, \mathscr{Y}}^{\oplus}\left[\mathscr{S}_{\omega}^{\perp} \cap E\right] d \mu(\omega)$,

5. $\operatorname{Ref}_{\mathscr{E}}\left(\int_{\Omega, \mathscr{L}}^{\oplus} \mathscr{S}_{\omega} d \mu(\omega)\right)=\int_{\Omega, \mathscr{L}}^{\oplus} \operatorname{Ref}_{E}\left(\mathscr{S}_{\omega}\right) d \mu(\omega)$, and

6. $\int_{\Omega, \mathscr{L}}^{\oplus} \mathscr{S}_{\omega} d \mu(\omega)$ is $\mathscr{E}$-reflexive if and only if $\mathscr{S}_{\omega}$ is E-reflexive a.e. $(\mu)$.

Proof. (1). It follows from (ii) above and the $\sigma$-finiteness of $\mu$ that there is a disjoint sequence $\left\{A_{n}\right\}$ of measurable subsets of finite measure with

$$
\mu\left(\Omega \backslash \bigcup_{n \geq 1} A_{n}\right)=0,
$$

and a sequence $\left\{u_{n}\right\}$ in $\mathscr{X}$ such that $u_{n}(\omega) \neq 0$ for $\omega \in A_{n}$ and $n \geq 1$. Since $\left\{S_{\omega}: \omega \in \Omega\right\}$ is measurable, there is a sequence $\left\{v_{m}\right\}$ of measurable functions from $\Omega$ into $X$ such that $\mathscr{S}_{\omega}=\left\{v_{m}(\omega): m \geq 1\right\}^{-}$a.e. $(\mu)$. Clearly, the sequence $\left\{f_{k}\right\}$ of all functions of the form

$$
\omega \mapsto r \chi_{A_{n}}(\omega) v_{m}(\omega)\left[\left\|u_{n}(\omega)\right\| /\left(1+\left\|v_{m}(\omega)\right\|\right)\right],
$$

with $m, n \geq 1$ and $r$ a positive rational number, satisfies the desired condition in statement (1) above.

(2) It is clear from the definition of direct integrals that if $\mathscr{S}_{\omega}=\mathscr{T}_{\omega}$ a.e. $(\mu)$, then $\int_{\Omega, \mathscr{L}}^{\oplus} \mathscr{S}_{\omega} d \mu(\omega)=\int_{\Omega, \mathscr{L}}^{\oplus} \mathscr{T}_{\omega} d \mu(\omega)$. It follows from (1) that we can choose sequences $\left\{u_{n}\right\}$ and $\left\{v_{n}\right\}$ in $\mathscr{X}$ so that $\mathscr{S}_{\omega}=\left\{u_{n}(\omega): n \geq 1\right\}^{-}$and $\mathscr{T}_{\omega}=\left\{v_{n}(\omega): n \geq 1\right\}^{-}$a.e. $(\mu)$. If $\int_{\Omega, \mathscr{L}}^{\oplus} \mathscr{S}_{\omega} d \mu(\omega)=\int_{\Omega, \mathscr{L}}^{\oplus} \mathscr{T}_{\omega} d \mu(\omega)$, then $v_{n} \in \int_{\Omega, \mathscr{L}}^{\oplus} \mathscr{S}_{\omega} d \mu(\omega)$ and $u_{n} \in \int_{\Omega, \mathscr{L}}^{\oplus} \mathscr{T}_{\omega} d \mu(\omega)$ for $n \geq 1$. It follows that $\mathscr{S}_{\omega}=\mathscr{\mathscr { T }}_{\omega}$ a.e. $(\mu)$.

(3) It is clear that $\left(\int_{\Omega, \mathscr{L}}^{\oplus} \mathscr{S}_{\omega} d \mu(\omega)\right)^{\perp}$ contains $\int_{\Omega, \mathscr{Y}}^{\oplus} \mathscr{S}_{\omega}^{\perp} d \mu(\omega)$. Suppose that

$$
g \in\left(\int_{\Omega, \mathscr{L}}^{\oplus} \mathscr{S}_{\omega} d \mu(\omega)\right)^{\perp},
$$

and, by (1), choose a sequence $\left\{u_{n}\right\}$ in $\int_{\Omega, \mathscr{X}}^{\oplus} \mathscr{S}_{\omega} d \mu(\omega)$ such that $\mathscr{S}_{\omega}=$ $\left\{u_{n}(\omega): n \geq 1\right\}^{-}$a.e. $(\mu)$. For each measurable subset $A$ of $\Omega$ and each positive integer $n$, we have $\chi_{A} u_{n} \in \int_{\Omega, p}^{\oplus} \mathscr{S}_{\omega} d \mu(\omega)$; whence,

$$
\int_{A}\left\langle u_{n}(\omega), g(\omega)\right\rangle d \mu(\omega)=0 .
$$


Thus $\left\langle u_{n}(\omega), g(\omega)\right\rangle=0$ a.e. $(\mu)$ for $n \geq 1$. Therefore, $g \in \int_{\Omega, y^{\oplus}}^{\oplus} \mathscr{S}_{\omega}^{\perp} d \mu(\omega)$.

(4) This follows from (3).

(5) It follows from (4) that

$$
\operatorname{Ref}_{\mathscr{C}}\left(\int_{\Omega, \mathscr{L}}^{\oplus} \mathscr{S}_{\omega} d \mu(\omega)\right)=\left(\int_{\Omega, \mathscr{Y}}^{\oplus}\left[\mathscr{S}_{\omega}^{\perp} \cap E\right] d \mu(\omega)\right)_{\perp} .
$$

It follows as in the proof of (3) that

$$
\left(\int_{\Omega, \mathscr{Y}}^{\oplus}\left[\mathscr{S}_{\omega}^{\perp} \cap E\right] d \mu(\omega)\right)_{\perp}=\int_{\Omega, \mathscr{Y}}^{\oplus}\left[\mathscr{S}_{\omega}^{\perp} \cap E\right]_{\perp} d \mu(\omega)=\int_{\Omega, \mathscr{L}}^{\oplus} \operatorname{Ref}_{E}\left(\mathscr{S}_{\omega}\right) d \mu(\omega) \text {. }
$$

(6) This follows from (1) and (5).

The following theorem is a generalization of [HN1, Theorem 3.6].

Theorem 3.5. Suppose $\left\{\mathscr{S}_{\omega}: \omega \in \Omega\right\}$ is a measurable family of $\sigma(X, Y)$-closed linear subspaces of $X$. If $Y$ is E-elementary of order $r$ on almost every $\mathscr{S}_{\omega}$, then $\mathscr{Y}$ is $\mathscr{E}$-elementary on $\int_{\Omega, \mathscr{L}}^{\oplus} \mathscr{S}_{\omega} d \mu(\omega)$. More precisely, for each $g \in \mathscr{Y}$, and each strictly positive measurable function $\psi$ on $\Omega$, there is an $e$ in $\mathscr{E}$ such that

(1) $g-e \in\left(\int_{\Omega, \mathscr{L}}^{\oplus} \mathscr{S}_{\omega} d \mu(\omega)\right)^{\perp}$, and

(2) $\|e(\omega)\| \leq[r+\psi(\omega)]\|\cdot\| g(\omega) \|$ a.e. $(\mu)$.

Proof. We can assume that $\psi \leq 1$; otherwise, replace $\psi$ with $\min (\psi, 1)$. Let $Q=\left\{(\omega, e) \in \Omega \times E: g(\omega)-e \in \mathscr{S}_{\omega}^{\perp},\|e\| \leq(r+\psi(\omega))\|g(\omega)\|\right\}$. Then $Q$ is a measurable subset of $\Omega \times E$ and therefore, by Proposition 3.1, $\pi_{1}$ has an absolutely measurable cross-section $\gamma$. Since $Y$ is $E$-elementary of order $r$ on almost every $\mathscr{S}_{\omega}$, we know that, by removing a set of measure 0 , we can assume that $\pi_{1}(Q)=\Omega$. Define a measurable map $e: \Omega \rightarrow E$ by $e=\pi_{2} \circ \gamma$. Statement (2) is now obvious, and it follows from (2) and the boundedness of $\psi$ that $e \in \mathscr{E}$. Furthermore it is evident from part (3) of Theorem 3.4 that statement (1) holds.

For the remainder of this section we will need norms on $\mathscr{Z}$ and on $\mathscr{Y}$. We will assume that

(Bvi) there is a norm \|\| on $\mathscr{Z}$ such that $\|u\| \leq\|v\|$ whenever $u, v \in \mathscr{Z}$ and $\|u(\omega)\| \leq\|v(\omega)\|$ a.e. $(\mu)$,

(Bvii) for each $g$ in $\mathscr{Y}$, the map $f \mapsto\langle f, g\rangle$ is norm continuous on $\mathscr{X}$.

We give $\mathscr{Y}$ the norm obtained from the embedding of $\mathscr{Y}$ into the dual space of $\mathscr{Z}$. In this case we have

(Bviii) $\|g\| \leq\|h\|$ whenever $g, h \in \mathscr{Y}$ and $\|g(\omega)\| \leq\|h(\omega)\|$ a.e. $(\mu)$, and (Bix) $|\langle f, g\rangle| \leq\|f\|\|g\|$ for all $f \in \mathscr{X}$ and $g \in \mathscr{Y}$.

A natural example of such norm arises when $\mathscr{X}=L_{Y}^{p}(\mu, X)$ and $\mathscr{Y}=$ $L_{X}^{q}(\mu, Y)$. In this case, with the added restriction that $p=\infty$, the following corollary is a generalization of [HN1, Theorem 3.6].

Corollary 3.6. Suppose $\left\{\mathscr{S}_{\omega}: \omega \in \Omega\right\}$ is a measurable family of $\sigma(X, Y)$-closed linear subspaces of $X$. If $Y$ is E-elementary of order $r$ on almost every $\mathscr{S}_{\omega}$, then $\mathscr{Y}$ is $\mathscr{E}$-elementary of order $r$ on $\int_{\Omega, \mathscr{D}}^{\oplus} \mathscr{S}_{\omega} d \mu(\omega)$.

The following is a generalization of the main result in [HN2]. The proof is essentially the same as the one in [HN2] and is omitted. 
Theorem 3.7. Suppose that $\left\{\mathscr{S}_{\omega}: \omega \in \Omega\right\}$ is a measurable family of $\sigma(X, Y)$ closed linear subspaces of $X$, and $\mathscr{S}=\int_{\Omega, p}^{\oplus} \mathscr{S}_{\omega} d \mu(\omega)$. Suppose that, for every sequence $\left\{f_{n}\right\}$ in $\mathscr{Y}$ such that $\sum_{n}\left\|f_{n}\right\|<\infty$ and the sets $\Omega_{n}=\left\{\omega: f_{n}(\omega) \neq\right.$ $0\}$ form a disjoint collection, there is an $f$ in $\mathscr{Y}$ such that, for each $n$, $\left(f-f_{n}\right) \mid \Omega_{n}=0$ a.e. $(\mu)$. If $\mu$ is a nonatomic and $\mathscr{Y}$ is $\mathscr{E}$-elementary on $\mathscr{S}$, then there is an $r>0$ such that $\mathscr{Y}$ is $\mathscr{E}$-elementary of order $r$ on $\mathscr{S}$.

The following is the main result on hyperreflexivity and direct integrals.

Theorem 3.8. Suppose $\left\{\mathscr{S}_{\omega}: \omega \in \Omega\right\}$ is a measurable family of $\sigma(X, Y)$-closed linear subspaces of $X$, and $\mathscr{S}=\int_{\Omega, \mathscr{L}}^{\oplus} \mathscr{S}_{\omega} d \mu(\omega)$. Then

(1) If $f \in \mathscr{X}$, then $d_{Y}\left(f(\omega), \mathscr{S}_{\omega}\right)$ and $d_{E}\left(f(\omega), \mathscr{S}_{\omega}\right)$ are measurable functions on $\Omega$.

(2) If $f \in \mathscr{Z}$, then

$$
d_{\mathscr{Y}}(f, \mathscr{S})=\sup \left\{\int_{\Omega} d_{Y}\left(f(\omega), \mathscr{S}_{\omega}\right)\|g(\omega)\| d \mu(\omega): g \in \operatorname{ball}(\mathscr{Y})\right\} .
$$

(3) If $f \in \mathscr{X}$, then

$$
d_{\mathscr{E}}(f, \mathscr{S})=\sup \left\{\int_{\Omega} d_{E}\left(f(\omega), \mathscr{S}_{\omega}\right)\|g(\omega)\| d \mu(\omega): g \in \operatorname{ball}(\mathscr{Y})\right\} .
$$

(4) The function $\omega \mapsto K_{E}\left(\mathscr{S}_{\omega}\right)$ is measurable on $\Omega$.

$$
K_{\mathscr{E}}\left(\int_{\Omega, p}^{\oplus} \mathscr{S}_{\omega} d \mu(\omega)\right)=\operatorname{ess}-\sup K_{E}\left(\mathscr{S}_{\omega}\right)
$$

(6) $\int_{\Omega, \mathscr{L}}^{\oplus} \mathscr{S}_{\omega} d \mu(\omega)$ is $\mathscr{E}$-hyperreflexive if and only if almost every $\mathscr{S}_{\omega}$ is $E$-hyperreflexive and ess-sup $K_{E}\left(\mathscr{S}_{\omega}\right)<\infty$.

Proof. (1) Suppose $f \in \mathscr{X}$. Choose sequences $\left\{u_{n}\right\}$ and $\left\{v_{n}\right\}$ of measurable functions on $\Omega$ such that $\left\{u_{n}(\omega): n \geq 1\right\}$ is dense in ball $\left(\mathscr{S}_{\omega}^{\perp}\right)$ and $\left\{v_{n}(\omega): n \geq 1\right\}$ is dense in $\mathscr{S}_{\omega}^{\perp} \cap \widetilde{E}$ a.e. $(\mu)$. Then

$$
d_{Y}\left(f(\omega), \mathscr{S}_{\omega}\right)=\sup _{n \geq 1}\left|\left\langle f(\omega), u_{n}(\omega)\right\rangle\right|
$$

and $d_{E}\left(f(\omega), \mathscr{S}_{\omega}\right)=\sup _{n \geq 1}\left|\left\langle f(\omega), v_{n}(\omega)\right\rangle\right|$ a.e. $(\mu)$.

(2) Suppose $f \in \mathscr{X}$. Let $\beta$ denote the right-hand side of the equation in (2) above. If $g \in \operatorname{ball}\left(\mathscr{S}^{\perp}\right)$, then $g(\omega) \in \mathscr{S}_{\omega}^{\perp}$ a.e. $(\mu)$. Thus $|\langle f, g\rangle| \leq$ $\int_{\Omega}|\langle f(\omega), g(\omega)\rangle| d \mu(\omega) \leq \int_{\Omega} d_{Y}\left(f(\omega), \mathscr{S}_{\omega}\right)\|g(\omega)\| d \mu(\omega) \leq \beta$. Hence $d_{\mathscr{y}}(f, \mathscr{S}) \leq \beta$. On the other hand, suppose $g \in$ ball(y) and $\varepsilon>0$. Choose a strictly positive $\psi$ in $L^{1}(\mu)$ so that $\|\psi\|_{1}=\varepsilon$. Let $Q=\{(\omega, h) \in$ $\left.\left.\Omega \times \operatorname{ball}(Y): h(\omega) \in \operatorname{ball}\left(\mathscr{S}_{\omega}^{\perp}\right),\left[d_{Y}\left(f(\omega), \mathscr{S}_{\omega}\right)-\psi(\omega)\right] \leq\langle f(\omega), h)\right\rangle\right\}$. Then $Q$ is a measurable subset of $\Omega \times Y$ and, by Proposition 3.1, the coordinate projection $\pi_{1}$ on $Q$ has a measurable cross-section $\alpha$. Note that $\pi_{1}(Q)=\Omega$ a.e. $(\mu)$. Let $h(\omega)=\pi_{2}(\alpha(\omega))\|g(\omega)\|$. Then $h \in$ ball $\left(\mathscr{S}^{\perp}\right)$ and

$$
\begin{aligned}
|\langle f, h\rangle| & =\int_{\Omega}\langle f(\omega), h(\omega)\rangle d \mu(\omega) \geq \int_{\Omega}\left[d_{Y}\left(f(\omega), \mathscr{S}_{\omega}\right)-\psi(\omega) /\|g(\omega)\|\right] d \mu(\omega) \\
& =\int_{\Omega} d_{Y}\left(f(\omega), \mathscr{S}_{\omega}\right)\|g(\omega)\| d \mu(\omega)-\|\psi\|_{1} .
\end{aligned}
$$


Hence we have

$$
\int_{\Omega} d_{Y}\left(f(\omega), \mathscr{S}_{\omega}\right)\|g(\omega)\| d \mu(\omega) \leq|\langle f, h\rangle|+\varepsilon \leq d_{\mathscr{Y}}(f, \mathscr{S})+\varepsilon .
$$

Since $\varepsilon>0$ was arbitrary, we see that $\beta \leq d_{y}(f, \mathscr{S})$.

(3) The proof is the same as that of (2) with $\mathscr{S}_{\omega}^{\perp} \cap \widetilde{E}$ replacing ball $\left(\mathscr{S}_{\omega}^{\perp}\right)$ and $\mathscr{S}^{\perp} \cap \mathscr{E}$ replacing ball $\left(\mathscr{S}^{\perp}\right)$.

(4) We first show that the map $s: \Omega \times \operatorname{ball}(X) \rightarrow[0, \infty)$ defined by $s(\omega, x)=$ $d_{Y}\left(x, \mathscr{S}_{\omega}\right)$ is measurable. To see this, we choose a sequence $\left\{g_{n}\right\}$ of measurable functions on $\Omega$ so that $\left\{g_{n}(\omega): n \geq 1\right\}$ is dense in ball $\left(\mathscr{S}_{\omega}^{\perp}\right)$ a.e. $(\mu)$, and note that $s(\omega, x)=\sup _{n>1}\left|\left\langle x, g_{n}(\omega)\right\rangle\right|$ a.e. $(\mu)$. A similar argument shows that the function $t: \Omega \times \operatorname{ball}(X) \rightarrow[0, \infty)$ defined by $t(\omega, x)=d_{E}\left(x, \mathscr{S}_{\omega}\right)$ is measurable. Note that $t \leq s$. Suppose $r>0$ and define $Q_{r}=\{(\omega, x) \in$ $\Omega \times \operatorname{ball}(X): s(\omega, x)>r \cdot t(\omega, x)\}$. Then $Q_{r}$ is measurable and $\pi_{1}\left(Q_{r}\right) \in \mathscr{M}$. However, $\pi_{1}\left(Q_{r}\right)=\left\{\omega \in \Omega: \exists x \in \operatorname{ball}(X), d_{Y}\left(x, \mathscr{S}_{\omega}\right)>\operatorname{rd}_{E}\left(x, \mathscr{S}_{\omega}\right)\right\}=\{\omega \in$ $\left.\Omega: K_{E}\left(\mathscr{S}_{\omega}\right)>r\right\}$ a.e. $(\mu)$. This proves $(4)$.

(5) It follows from (2) and (3) that $K_{\mathscr{E}}(\mathscr{S}) \leq \operatorname{ess-sup} K_{E}\left(\mathscr{S}_{\omega}\right)$. On the other hand, suppose $r<\operatorname{ess}-\sup K_{E}\left(\mathscr{S}_{\omega}\right)$. It follows that if $Q_{r}$ is defined as in the proof of (4), then $\mu\left(\pi_{1}\left(Q_{r}\right)\right)>0$. We also know from the defining assumptions on $\mathscr{X}$ that there is a subset $A$ of $\pi_{1}\left(Q_{r}\right)$ with $\mu(A)>0$ and a function $h$ in $\mathscr{X}$ such that $h(\omega) \neq 0$ for every $\omega$ in $A$. We know from Proposition 3.1 that the map $\pi_{1}: Q_{r} \rightarrow \Omega$ has an absolutely measurable cross-section $\alpha$. We define $f: \Omega \rightarrow X$ by $f(\omega)=0$ off $A$ and $f(\omega)=$ $\pi_{2}(\alpha(\omega))\left[\|h(\omega)\| /\left\|\pi_{2}(\alpha(\omega))\right\|\right]$ on $A$. Then $f \in \mathscr{X}$, and it follows from (2) and (3) above that $d_{\mathscr{y}}(f, \mathscr{S})>r d_{\mathscr{E}}(f, \mathscr{S})$. Hence, $r \leq K_{\mathscr{E}}(\mathscr{S})$. Since $r$ was arbitrary, it follows that ess-sup $K_{E}\left(\mathscr{S}_{\omega}\right) \leq K_{\mathscr{C}}(\mathscr{S})$.

(6) This follows from (5).

Corollary 3.9. If, in the preceding theorem, $\mathscr{X}=L_{Y}^{p}(\mu, X)$ and $\mathscr{Y}=L_{Y}^{q}(\mu, Y)$, then, for each $f$ in $\mathscr{X}$, we have

(a) $d_{y}(f, \mathscr{S})$ is the $L^{p}$-norm of $d_{Y}\left(f(\omega), \mathscr{S}_{\omega}\right)$.

(b) $d_{\mathscr{E}}(f, \mathscr{S})$ is the $L^{p}$-norm of $d_{E}\left(f(\omega), \mathscr{S}_{\omega}\right)$.

\section{DiRECT SUMS}

Unlike the case of direct integrals, with direct sums there is no need for measurability considerations, so many restrictions can be removed. In this case, we can assume that $\mu$ is counting measure on an arbitrary set $\Omega$. Let $\left\{X_{\omega}: \omega \in\right.$ $\Omega\}$ be a collection of normed linear spaces. The algebraic direct product $\prod X_{\omega}$ of the $X_{\omega}$ 's is the set of all functions $f: \Omega \rightarrow \bigcup X_{\omega}$ such that $f(\omega) \in X_{\omega}$ of every $\omega$ in $\Omega$. If $f \in \prod X_{\omega}$, then the support of $f$ is the $\operatorname{set} \operatorname{supp}(f)=$ $\{\omega \in \Omega: f(\omega) \neq 0\}$. The algebraic direct sum $\sum X_{\omega}$ of the $X_{\omega}$ 's is the set of all functions in $\prod X_{\omega}$ with finite support. We say that a vector space $\mathscr{Z}$ is a direct sum of the $X_{\omega}$ 's if

(1) $\sum X_{\omega} \subset \mathscr{X} \subset \prod X_{\omega}$,

(2) if $f \in \mathscr{X}$ and $g \in \prod X_{\omega}$ and $\|g(\omega)\| \leq\|f(\omega)\|$ for every $\omega$ in $\Omega$, then $g \in \mathscr{X}$.

We say that $\mathscr{X}$ is a normed direct sum if there is a norm \|\| on $\mathscr{X}$ such that $\|g\| \leq\|f\|$ whenever $f, g \in \mathscr{X}$ and $\|g(\omega)\| \leq\|f(\omega)\|$ for every $\omega$ in $\Omega$, and such that $\|f(\omega)\| \leq\|f\|$ for every $f$ in $\mathscr{X}$ and every $\omega$ in $\Omega$. 
Suppose that $A_{\omega} \subset X_{\omega}$ for each $\omega$ in $\Omega$. We define the direct sum of the $A_{\omega}$ 's relative to $\mathscr{Z}$, denoted $\sum_{\mathscr{X}}^{\oplus} A_{\omega}$, as the set of all $f$ in $\mathscr{Z}$ such that $f(\omega) \in A_{\omega}$ for every $\omega$ in $\Omega$.

Suppose, for each $\omega$ in $\Omega$, that $\left(X_{\omega}, Y_{\omega}, E_{\omega}\right)$, is a normed reflexivity triple. We say that $(\mathscr{X}, \mathscr{Y}, \mathscr{E})$ is a direct sum of the $\left(X_{\omega}, Y_{\omega}, E_{\omega}\right)$ 's if

(3) $\mathscr{X}$ is a direct sum of the $X_{\omega}$ 's,

(4) $\mathscr{Y}$ is a direct sum of the $Y_{\omega}$ 's,

(5) $\mathscr{E}=\sum_{\mathscr{Y}}^{\oplus} E_{\omega}$, and

(6) $\sum|\langle f(\omega), h(\omega)\rangle|<\infty$ for every $f$ in $\mathscr{X}$ and every $h$ in $\mathscr{Y}$.

By $(6),\langle f, h\rangle=\sum\langle f(\omega), h(\omega)\rangle$ defines a duality between $\mathscr{Z}$ and $\mathscr{Y}$ that makes $(\mathscr{Z}, \mathscr{Y}, \mathscr{E})$ a reflexivity triple.

We say that $(\mathscr{X}, \mathscr{Y}, \mathscr{E})$ is a normed direct sum of the $\left(X_{\omega}, Y_{\omega}, E_{\omega}\right)$ 's if, in addition to (3)-(6), $\mathscr{Z}$ is a normed direct sum of the $X_{\omega}$ 's, and, with the duality given by (6), $\mathscr{Y} \subset \mathscr{X}^{\#}$. It is clear, with the norm on $\mathscr{Y}$ induced by $\mathscr{Z}^{\#}$, that $\mathscr{Y}$ is a normed direct sum of the $Y_{\omega}$ 's.

Most of the main results in the preceding section have analogues for direct sums. The proofs are all straightforward and are omitted.

Theorem 4.1. Suppose $(\mathscr{X}, \mathscr{Y}, \mathscr{E})$ is a direct sum of a family $\left\{\left(X_{\omega}, Y_{\omega}, E_{\omega}\right)\right.$ : $\omega \in \Omega\}$ of reflexivity triple. Suppose, for each $\omega$ in $\Omega$, that $\mathscr{S}_{\omega}$ is a linear subspace of $X_{\omega}$, and that $\mathscr{S}=\sum_{\mathscr{X}}^{\oplus} \mathscr{S}_{\omega}$. Then

(1) $\operatorname{Ref}_{\mathscr{E}}(\mathscr{S})=\sum_{\mathscr{X}}^{\oplus} \operatorname{Ref}_{E}\left(\mathscr{S}_{\omega}\right)$,

(2) $\mathscr{S}$ is $\mathscr{E}$-reflexive if and only if every $\mathscr{S}_{\omega}$ is $E_{\omega}$-reflexive, and

(3) if $r \geq 1$ and, for every $\omega$ in $\Omega, Y_{\omega}$ is $E_{\omega}$-elementary of order $r$ on $\mathscr{S}_{\omega}$, then $\mathscr{Y}$ is $\mathscr{E}$-elementary on $\mathscr{S}$. have

Moreover, if $(\mathscr{X}, \mathscr{Y}, \mathscr{E})$ is a normed direct sum of the $\left(X_{\omega}, Y_{\omega}, E_{\omega}\right)$ 's, we

(4) if $r \geq 1$ and, for every $\omega$ in $\Omega, Y_{\omega}$ is $E_{\omega}$-elementary of order $r$ on $\mathscr{S}_{\omega}$, then $\mathscr{Y}$ is $\mathscr{E}$-elementary of order $r$ on $\mathscr{S}$,

(5) $K_{\mathscr{E}}(\mathscr{S})=\sup _{\omega} K_{E}\left(\mathscr{S}_{\omega}\right)$,

(6) $\mathscr{S}$ is $\mathscr{E}$-hyperreflexive if and only if every $\mathscr{S}_{\omega}$ is $E_{\omega}$-hyperreflexive and $\sup _{\omega} K_{E}\left(\mathscr{S}_{\omega}\right)<\infty$.

Combining the results in this section with those in the preceding section, we can define a more general notion of "direct integral" that is formed by taking direct sums of direct integrals. This allows us to handle the usual direct integral theory in Hilbert spaces [Dix].

\section{Applications}

\section{Algebraic REFLeXIVITY}

In this section we apply the results in $\S 1$ in a purely algebraic setting. Suppose $V$ and $W$ are vector spaces over a field $F$ with the discrete topology. We let $\mathscr{L}(V, W)$ denote the set of $F$-linear transformations from $V$ to $W$, we let $\mathscr{L}(V)=\mathscr{L}(V, V)$, and we let $V^{\prime}$ denote $\mathscr{L}(V, F)$ (the algebraic dual of $V$ ). If $v \in V$ and $\alpha \in W^{\prime}$, we define the rank-one-tensor $v \otimes \alpha$ in $\mathscr{L}(V, W)^{\prime}$ by $(v \otimes \alpha)(T)=\alpha(T v)$. Throughout this section $X=\mathscr{L}(V, W), E=\{v \otimes \alpha: v \in$ $\left.V, \alpha \in W^{\prime}\right\}$ and $E \subset Y \subset L(V, W)^{\prime}$.

If $\mathscr{S}$ is a linear subspace of $\mathscr{L}(V, W)$, we let $\operatorname{Ref}_{0}(\mathscr{S})=\operatorname{Ref}_{E}(\mathscr{S})$, and 
we say that $\mathscr{S}$ is algebraically reflexive if $\mathscr{S}$ is $E$-reflexive. The following lemma is obvious, but it points out the traditional way of viewing algebraic reflexivity.

Lemma 5.1. Suppose that $\mathscr{S}$ is a linear subspace of $\mathscr{L}(V, W)$ and $T \in$ $\mathscr{L}(V, W)$. Then $T \in \operatorname{Ref}_{0}(\mathscr{S})$ if and only if, for each $v$ in $V$, we have $T v \in \mathscr{S} v$.

In the case in which $V=W$ and $\mathscr{S}$ is a unital algebra, algebraic reflexivity can be defined in terms of invariant linear manifolds. If $\mathscr{S} \subset \mathscr{L}(V)$, then $\operatorname{Lat}_{0}(\mathscr{S})$ is the set of all linear subspaces $M$ of $V$ such that, for every $S$ in $\mathscr{S}$, we have $S(M) \subset M$. We define $\operatorname{Alg}_{\operatorname{Lat}}(\mathscr{S})$ to be $\left\{T \in \mathscr{L}(V): \operatorname{Lat}_{0}(\mathscr{S}) \subset\right.$ $\left.\operatorname{Lat}_{0}(T)\right\}$.

Lemma 5.2. If $\mathscr{S}$ is a unital subalgebra of $\mathscr{L}(V)$, then $\operatorname{Ref}_{0}(\mathscr{S})=\operatorname{Alg}_{\operatorname{Lat}}(\mathscr{S})$.

Even in the purely algebraic setting, there is a natural topology on $\mathscr{L}(V, W)$, namely the strict topology. For each finite subset $G$ of $V$ and each $T$ in $\mathscr{L}(V, W)$, we have a basic strict neighborhood $U(T, G)$ of $T$ defined by $U(T, G)=\left\{S \in \mathscr{L}(V, W):\left.S\right|_{G}=\left.T\right|_{G}\right\}$. A net $\left\{T_{n}\right\}$ in $\mathscr{L}(V, W)$ is strictly convergent to a transformation $T$ if and only if, for each $v$ in $V$ we have eventually that $T_{n} v=T v$. Hence the strict topology may be viewed as the topology of pointwise convergence with respect to the discrete topology on $W$.

The smallest possible choice for $Y$ is $\mathrm{sp} E$, the set of finite linear combinations of rank-one tensors. These functionals arise naturally in terms of the strict topology.

Lemma 5.3. Suppose $\varphi$ is a linear functional in $\mathscr{L}(V, W)^{\prime}$. The following are equivalent.

(1) $\varphi$ is a finite linear combination of rank-one tensors,

(2) $\varphi$ is continuous with respect to the strict topology on $\mathscr{L}(V, W)$ and the discrete topology on $F$,

(3) $\operatorname{ker} \varphi$ is strictly closed,

(4) $\operatorname{ker} \varphi$ is strictly open,

(5) there is a finite subset $G$ of $V$ such that $\{T \in \mathscr{L}(V, W): T \mid G=0\} \subset$ $\operatorname{ker} T$.

Proof. The implications (1) $\Rightarrow(2) \Rightarrow(3)$ and (4) $\Leftrightarrow(5)$ are obvious; (5) $\Rightarrow(1)$ follows as in standard duality theory by factoring $\varphi$ through a finite direct sum of copies of $W$. To prove $(3) \Rightarrow(5)$, assume $\varphi \neq 0$ and $\operatorname{ker} \varphi$ is strictly closed. Suppose $T \notin \operatorname{ker} \varphi$. Hence there is a finite subset $G$ of $V$ such that $U(T, G) \cap \operatorname{ker} \varphi=\varnothing$. Hence if $S \in \mathscr{L}(V, W)$ and $S \mid G=0$, then, for every $\lambda \in F$, we have $T-\lambda S \in U(T, G)$, which implies that $\varphi(T) \neq \lambda \varphi(S)$. It follows that $S \mid G=0$ implies $\varphi(S)=0$. Thus (5) is true.

Corollary 5.4. If $Y=\operatorname{sp} E$, and $\mathscr{S}$ is a linear subspace of $\mathscr{L}(V, W)$, then $\mathscr{S}$ is $\sigma(X, Y)$-closed if and only if $\mathscr{S}$ is strictly closed.

Note that in the case in which $Y$ is all of $\mathscr{L}(V, W)^{\prime}$, we have that every linear subspace of $\mathscr{L}(V, W)$ is $\sigma(X, Y)$-closed. Hence we have two distinct versions of the Loginov-Shulman theorem (Theorem 2.1(3)) in the algebraic setting. 
Theorem 5.5. Suppose $\mathscr{S}$ is an algebraically reflexive linear subspace of $\mathscr{L}(V, W)$. Then the following are true.

(1) Every linear subspace of $\mathscr{S}$ is algebraically reflexive if and only if every linear functional on $\mathscr{L}(V, W)$ can be represented on $\mathscr{S}$ by a rank-one tensor.

(2) Every strictly closed linear subspace of $\mathscr{S}$ is algebraically reflexive if and only if each finite linear combination of rank-one tensors can be represented on $\mathscr{S}$ by a single rank-one tensor.

Example. Suppose $V=W$ and $B$ is a linear basis (i.e., Hamel basis) for $V$. Let $\mathscr{S}$ be the set of all linear transformations that are diagonal with respect to $B$, i.e., those transformations for which each vector in $B$ is an eigenvector. It is clear that $\mathscr{S}$ is algebraically reflexive. Moreover, if $v_{1}, v_{2}, \ldots, v_{n} \in V$ and $\alpha_{1}, \alpha_{2}, \ldots, \alpha_{n} \in V^{\prime}$, then, since each $v_{k}$ is a linear combination of finitely many elements of $B$, it follows that $\varphi=\sum_{k=1}^{n} v_{k} \otimes \alpha_{k}$ can be re-represented so that $v_{1}, v_{2}, \ldots, v_{n}$ are $n$ distinct elements in $B$. It is clear that if $\varphi$ is so represented, then $\varphi$ can be represented on $\mathscr{S}$ as $\left(\sum_{k=1}^{n} \alpha_{k}\left(v_{k}\right) v_{k}\right) \otimes \alpha$, where $\alpha$ is any functional that takes the value 1 at each $v_{k}$. Hence, by the preceding theorem, every strictly closed linear subspace of $\mathscr{S}$ is algebraically reflexive.

On the other hand, if $B$ is infinite, then the subspace $\mathscr{F}$ of finite-rank linear transformations in $\mathscr{S}$ is not algebraically reflexive; in fact, $\operatorname{Ref}_{0}(\mathscr{F})=\mathscr{S}$.

Remark. It may seem unlikely that a linear subspace $\mathscr{S}$ of $\mathscr{L}(V, W)$ has the property that every functional in $\mathscr{L}(V, W)^{\prime}$ can be represented on $\mathscr{S}$ as a rank-one tensor. However, this is the case when $\mathscr{S}$ has a separating vector $e$, that is, the map $\tau(S)=S e$ is 1-1 on $\mathscr{S}$. In this case, given a $\varphi$ in $\mathscr{L}(V, W)^{\prime}$, choose $\alpha \in W^{\prime}$ so that $\alpha \mid \mathscr{S}_{e}=\varphi \circ \tau^{-1}$; clearly, $\varphi$ agrees with $e \otimes \alpha$ on $\mathscr{S}$. The importance of separating vectors in algebraic reflexivity is demonstrated in [L3 and H2].

To see how to apply part (2) of Theorem 1.2 we must use the notion of relative reflexivity. Suppose $n$ is a positive integer and $T \in \mathscr{L}(V, W)$. We let $V^{(n)}$ denote a direct sum of $n$ copies of $V$, and we define $T^{(n)}$ in $\mathscr{L}\left(V^{(n)}, W^{(n)}\right)$ by $T^{(n)}\left(v_{1}, v_{2}, \ldots, v_{n}\right)=\left(T_{v_{1}}, T_{v_{2}}, \ldots, T_{v_{n}}\right)$. If $\mathscr{S} \subset \mathscr{L}(V, W)$, we define $\mathscr{S}^{(n)}=\left\{T^{(n)}: T \in \mathscr{S}\right\}$. More generally, if $T_{1}, T_{2}, \ldots, T_{n} \in \mathscr{L}(V, W)$, we define $\left(T_{1} \oplus T_{2} \oplus \cdots \oplus T_{n}\right)$ in $\mathscr{L}\left(V^{(n)}, W^{(n)}\right)$ by $\left(T_{1} \oplus T_{2} \oplus \cdots \oplus T_{n}\right)\left(v_{1}, v_{2}, \ldots, v_{n}\right)$ $=\left(T_{1} v_{1}, \ldots, T_{n} v_{n}\right)$, and if $\mathscr{S}_{1}, \mathscr{S}_{2}, \ldots, \mathscr{S}_{n} \subset \mathscr{L}(V, W)$, we define $\mathscr{S}_{1} \oplus$ $\mathscr{S}_{2} \oplus \cdots \oplus \mathscr{S}_{n}=\left\{T_{1} \oplus T_{2} \oplus \cdots \oplus T_{n}: T_{k} \in \mathscr{S}_{k}\right\}$. It is easily shown that $\mathscr{L}(V, W)^{(n)}$ is always algebraically reflexive. Hence, to show that $\mathscr{S}^{(n)}$ is algebraically reflexive, it is necessary and sufficient to show that $\mathscr{S}^{(n)}$ is relatively algebraically reflexive with respect to $\mathscr{L}(V, W)^{(n)}$ (Theorem 2.6(1)).

Lemma 5.6. If $\mathscr{S}$ is a linear subspace of $\mathscr{L}(V, W)$ and $n$ is a positive integer, then $\operatorname{Ref}_{0}\left(\mathscr{S}^{(n)}\right)=\left[\operatorname{Ref}_{E_{n}}(\mathscr{S})\right]^{(n)}$. In particular, $\mathscr{S}^{(n)}$ is algebraically reflexive if and only if $\mathscr{S}$ is $E_{n}$-reflexive.

The following corollary is an interpretation of Theorem 1.2(4).

Corollary 5.7. If $\mathscr{S}$ is a linear subspace of $\mathscr{L}(V, W)$ and $T \in \mathscr{L}(V, W)$, then $T$ is in the strict closure of $\mathscr{S}$ if and only if, for each positive integer $n$, $T^{(n)} \in \operatorname{Ref}_{0}\left(\mathscr{S}^{(n)}\right)$.

The algebraic interpretation of part (2) of Theorem 1.2 is the following result. 
Proposition 5.8. Suppose $\mathscr{S}$ is a strictly closed linear subspace of $\mathscr{L}(V, W)$ and every finite linear combination of rank-one tensors can be represented on $\mathscr{S}$ as a single rank-one tensor. Then $\mathscr{S}^{(3)}$ is algebraically reflexive.

Remark. If we combine the preceding proposition with the remark following Theorem 5.5, we conclude that if $\mathscr{S}$ is a strictly closed linear subspace of $\mathscr{L}(V, W)$ and $\mathscr{S}$ has a separating vector, then $\mathscr{S}^{(3)}$ is algebraically reflexive. However, it was shown by P. Fillmore [Fi] that $\mathscr{S}^{(2)}$ is algebraically reflexive whenever $\mathscr{S}$ has a separating vector.

The notion of relative reflexivity is also needed to interpret Theorem 1.3 concerning reflexive graphs. Suppose that $V_{1}, V_{2}, W_{1}$, and $W_{2}$ are vector spaces over $F$ and $V=V_{1} \oplus V_{2}$ and $W=W_{1} \oplus W_{2}$. It is easily seen that $\mathscr{L}\left(V_{1}, W_{1}\right) \oplus$ $\mathscr{L}\left(V_{2}, W_{2}\right)$ is an algebraically reflexive subalgebra of $\mathscr{L}(V, W)$. Moreover, a rank-one tensor $\varphi=\left(v_{1}, v_{2}\right) \otimes\left(\alpha_{1}, \alpha_{2}\right)$ acts on $\mathscr{L}\left(V_{1}, W_{1}\right) \oplus \mathscr{L}\left(V_{2}, W_{2}\right)$ by $\varphi\left(T_{1} \oplus T_{2}\right)=\left(v_{1} \otimes \alpha_{1}\right)\left(T_{1}\right)+\left(v_{2} \otimes \alpha_{2}\right)\left(T_{2}\right)$. From the preceding facts, it is now clear how Theorem 1.3 implies the following proposition.

Proposition 4.9. Suppose that $\mathscr{S}$ is an algebraically reflexive subspace of $\mathscr{L}\left(V_{1}, W_{1}\right)$ and $\rho: \mathscr{S} \rightarrow \mathscr{L}\left(V_{2}, W_{2}\right)$ is a linear map. Then

(1) if every strictly closed linear subspace of $\mathscr{S}$ is algebraically reflexive and if $\rho$ is continuous with respect to the strict topologies on $\mathscr{S}$ and $\mathscr{L}\left(V_{2}, W_{2}\right)$, then $\operatorname{graph}(\rho)$ is an algebraically reflexive linear subspace of $\mathscr{L}\left(V_{1} \oplus V_{2}, W_{1} \oplus W_{2}\right)$, and

(2) if every linear subspace of $\mathscr{S}$ is algebraically reflexive, then $\operatorname{graph}(\rho)$ is algebraically reflexive.

\section{TOPOLOGICAL REFLEXIVITY}

In this section we assume that $V$ and $W$ are locally convex topological vector spaces and that $F$ is either the real or complex field. We will let $X=B(V, W)$, the set of continuous linear transformations from $V$ to $W$, and we let $E$ be the set of rank-one tensors $v \otimes \alpha$ with $v \in V$ and $\alpha \in W^{\#}$ (the continuous linear functionals on $W$ ).

If $\mathscr{S}$ is a linear subspace of $B(V, W)$, we let $\operatorname{Ref}(\mathscr{S})=\operatorname{Ref}_{E}(\mathscr{S})$, and we say that $\mathscr{S}$ is (topologically) reflexive if $\mathscr{S}$ is $E$-reflexive. If $V=W$ and $\mathscr{S} \subset B(V)$, we define Lat $\mathscr{S}$ to be the set of all closed linear subspaces in $\operatorname{Lat}_{0} \mathscr{S}$, and we define $\operatorname{Alg} \operatorname{Lat} \mathscr{S}=\{T \in B(V)$ : Lat $\mathscr{S} \subset \operatorname{Lat}(T)\}$. The following lemma is easily proved and points out the traditional way of viewing reflexivity.

Lemma 6.1. Suppose that $\mathscr{S}$ is a linear subspace of $B(V, W)$ and $T \in B(V, W)$. The following are equivalent:

(1) $T \in \operatorname{Ref}(\mathscr{S})$,

(2) for each $v$ in $V$, we have $T v \in(\mathscr{S} v)^{-}$,

(3) $A T B=0$ for each $A \in B(W)$ and $B \in B(V)$ such that $A \mathscr{S} B=\{0\}$,

(4) $P T Q=0$ for each $P \in B(W)$ and $Q \in B(V)$ such that $P=P^{2}$, $Q=Q^{2}$, and $P \mathscr{S} Q=\{0\}$.

Moreover, if $V=W$ and $\mathscr{S}$ is a unital subalgebra of $B(V)$, then $\operatorname{Ref} \mathscr{S}=$ $\operatorname{Alg} \operatorname{Lat} \mathscr{S}$.

Remarks. 1. If $V$ and $W$ are normed spaces and $\varepsilon>0$, then Lemma 6.1 remains true when the conditions $\|P\|<1+\varepsilon,\|Q\|=1$, and $\operatorname{rank} P=$ 
rank $Q=1$ are added to part (4). If $V$ and $W$ are classically reflexive Banach spaces (i.e., canonically equal to their second duals), then Lemma 6.1 remains true when the conditions $\|P\|=\|Q\|=1=\operatorname{rank} P=$ rank $Q$ are added to part (4).

2. It follows from part (3) of the preceding lemma that $\operatorname{Ref}(\mathscr{S})$ is an algebra (resp. ring) whenever $\mathscr{S}$ is. In fact, more can be said. Suppose $\mathscr{R}$ is a semigroup and $\mathscr{S}$ is a subsemigroup of $\mathscr{R}$. Suppose $z \in \mathscr{R}$. Then $\mathscr{T}=$ $\{x \in \mathscr{R}: a x b=z$ whenever $a \mathscr{S} b=\{z\}\}$ is a subsemigroup of $\mathscr{R}$.

Proof. Suppose $x, y \in \mathscr{T}, a, b \in \mathscr{R}$, and $a \mathscr{S} b=\{z\}$. Since $\mathscr{S}$ is a subsemigroup, $a s \mathscr{S} b=\{z\}$ for every $s$ in $\mathscr{S}$. Since $y \in \mathscr{T}$, we conclude (as) $y b=z$ for every $s$ in $\mathscr{S}$; whence, $a \mathscr{S}(y b)=\{z\}$. But $x \in \mathscr{T}$; thus $a x(y b)=a(x y) b=z$. Hence $x y \in \mathscr{T}$, which shows that $\mathscr{T}$ is a semigroup.

As in the algebraic case, the smallest possible choice for $Y$ is $\operatorname{sp} E$, the set of finite linear combinations of rank-one tensors. The $\sigma(X, \mathrm{sp} E)$-topology is called the weak operator topology. There are many other possible topologies on $B(V, W)$. For example, if $V$ and $W$ are normed spaces, there is the norm topology, and we might choose $Y$ to be $B(V, W)^{\#}$.

If $V$ and $W$ are Banach spaces and $W$ is the normed dual $U^{\#}$ of a Banach space $U$, then $B(V, W)$ is the normed dual of the projective tensor product $V \otimes U$ (e.g., see [P]); in this case $B(V, W)$ has a weak *topology, and we might choose $Y=V \otimes U$. In order to ensure that $E \subset Y$, we must give $W$ the weak *-topology inherited from $U$. If $W$ is a classically reflexive Banach space, then $W^{\#}=U$ when $W$ is given the norm topology.

In the topological setting there are various possible versions of the LoginovShulman theorem (Theorem 1.2(4)).

Theorem 6.2. Suppose $\mathscr{S}$ is a reflexive linear subspace of $B(V, W)$. Then

(1) every weak-operator closed linear subspace of $\mathscr{S}$ is reflexive if and only if every weak-operator continuous linear functional on $B(V, W)$ can be represented on $\mathscr{S}$ by a rank-one tensor,

(2) when $V$ and $W$ are normed spaces, every norm close linear subspace of $\mathscr{S}$ is reflexive if and only if each norm continuous linear functional on $B(V, W)$ can be represented on $\mathscr{S}$ by a single rank-one tensor,

(3) when $U, V$, and $W$ are Banach spaces with $W=U^{\#}$, every weak*closed linear subspace of $\mathscr{S}$ is reflexive if and only if every functional in $V \otimes U$ can be represented on $\mathscr{S}$ as a rank-one tensor.

Remark. As in the algebraic case, it may seem unlikely that a linear subspace $\mathscr{S}$ of $B(V, W)$ has the property that every functional in $B(V, W)^{\#}$ can be represented on $\mathscr{S}$ as a rank-one tensor. However, if $V$ and $W$ are Banach spaces, then this is the case when $\mathscr{S}$ has a separating vector $e$ for which $\mathscr{S} e$ is norm closed in $W$. If $\tau: \mathscr{S} \rightarrow \mathscr{S} e$ is defined by $\tau(S)=S e$, then $\tau$ is continuous, 1-1 and onto, and it follows from the closed graph theorem that $\tau^{-1}$ is continuous. Hence, given a $\varphi$ in $B(V, W)^{\#}$, choose $\alpha \in W^{\#}$ so that $\alpha \mid \mathscr{S} e=\varphi \circ \tau^{-1}$; clearly, $\varphi$ agrees with $e \otimes \alpha$ on $\mathscr{S}$. These ideas appear in [HN1].

The results in the preceding section have natural analogues in the topological 
setting. If $V_{1}$ and $V_{2}$ are topological vector spaces, we always give $V_{1} \oplus V_{2}$ the product topology.

Proposition 6.3. Suppose $\mathscr{S}$ is a linear subspace of $B(V, W)$. Then

(1) if $n$ is a positive integer, then $\operatorname{Ref}\left(\mathscr{S}^{(n)}\right)=\left[\operatorname{Ref}_{E_{n}}(\mathscr{S})\right]^{(n)}$. In particular, $\mathscr{S}^{(n)}$ is reflexive if and only if $\mathscr{S}$ is $E_{n}$-reflexive.

(2) A transformation $T$ in $B(V, W)$ is in the weak-operator closure of $\mathscr{S}$ if and only if, for each positive integer $n, T^{(n)} \in \operatorname{Ref} \mathscr{S}^{(n)}$.

(3) If $\mathscr{S}$ is weak-operator closed and every weak-operator continuous linear functional can be represented on $\mathscr{S}$ as a single rank-one tensor, then $\mathscr{S}^{(3)}$ is reflexive.

The result of P. Fillmore [Fi] mentioned in the preceding section has an analogue in the topological case.

Lemma 6.4. Suppose $V$ and $W$ are Banach spaces, $\mathscr{S}$ is a norm closed linear subspace of $B(V, W)$ and $\mathscr{S}$ has a separating vector $e$ such that $\mathscr{S} e$ is norm closed in $W$. Then $\mathscr{S}^{(2)}$ is reflexive.

The techniques used in the preceding section on algebraically reflexive graphs carry over with few changes in the topological setting. Suppose that $V_{1}, V_{2}, W_{1}$, and $W_{2}$ are locally convex vector spaces over $F$ and $V=V_{1} \oplus V_{2}$ and $W=$ $W_{1} \oplus W_{2}$. It is easily seen that $B\left(V_{1}, W_{1}\right) \oplus B\left(V_{2}, W_{2}\right)$ is a reflexive subspace of $B(V, W)$. Hence we need only prove that $\operatorname{graph}(\rho)$ is relatively reflexive in $B\left(V_{1}, W_{1}\right) \oplus B\left(V_{2}, W_{2}\right)$ to prove the following proposition (see Theorem 2.6). However, the proposition now becomes an interpretation of Theorem 1.3.

Proposition 6.5. Suppose that $\mathscr{S}$ is a reflexive subspace of $B\left(V_{1}, W_{1}\right)$ and $\rho: \mathscr{S} \rightarrow B\left(V_{2}, W_{2}\right)$ is a linear map. The following are true.

(1) If every weak-operator closed linear subspace of $\mathscr{S}$ is reflexive and if $\rho$ is continuous with respect to the weak operator topologies on $\mathscr{S}$ and $B\left(V_{2}, W_{2}\right)$, then $\operatorname{graph}(\rho)$ is a reflexive linear subspace of $B\left(V_{1} \oplus V_{2}, W_{1} \oplus W_{2}\right)$.

(2) If every norm closed linear subspace of $\mathscr{S}$ is reflexive, then $\operatorname{graph}(\rho)$ is reflexive.

(3) If $V_{1}, V_{2}$ are Banach spaces and $W_{1}, W_{2}$ are dual Banach spaces and $\rho$ is continuous with respect to the weak ${ }^{*}$-topologies on $\mathscr{S}$ and $B\left(V_{2}, W_{2}\right)$, then $\operatorname{graph}(\rho)$ is reflexive.

For the remainder of this section, we assume that $V$ and $W$ are normed linear spaces. To interpret the notion of hyperreflexivity in our setting, we first examine the function $d_{E}$. If $M$ is a closed linear subspace of $V$, let $\pi_{M}: V \rightarrow V / M$ denote the canonical quotient map.

Lemma 6.6. Suppose $V$ and $W$ are normed spaces, $\mathscr{S}$ is a reflexive linear subspace of $B(V, W)$, and $T \in B(V, W)$. Then $d_{E}(T, \mathscr{S})$ equals each of the following:

(1) $\sup \{\operatorname{dist}(T v, \mathscr{S} v): v \in V,\|v\|=1\}$,

(2) $\sup \{\|A T B\|: A \in B(W), B \in B(V),\|A\|=\|B\|=1, A \mathscr{S} B=\{0\}\}$,

(3) $\lim _{\varepsilon \rightarrow 0^{+}} \sup \left\{\|P T Q\|: P^{2}=P \in B(W), Q^{2}=Q \in B(V),\|A\|,\|B\|<\right.$ $1+\varepsilon, A \mathscr{S} B=\{0\}\}$.

Moreover, if $V=W$ and $\mathscr{S}$ is a unital algebra, then

$$
d_{E}(T, \mathscr{S})=\sup \left\{\left\|\left.\pi_{M} T\right|_{M}\right\|: M \in \text { Lat } \mathscr{S}\right\} .
$$


Remark. In the case in which $V=W$ and $V$ is a Hilbert space, the closed linear subspaces of $V$ can be identified with the selfadjoint projections in $B(V)$ so that Lat $\mathscr{S}$ is the set of projections $P$ such that $(1-P) \mathscr{S} P=\{0\}$. If rank $P=M$, then $\|(1-P) T P\|=\left\|\left.\pi_{M} T\right|_{M}\right\|$. Thus if $\mathscr{S}$ is a unital subalgebra of $B(V)$, the preceding proposition asserts that

$$
d_{E}(T, \mathscr{S})=\sup \{\|(1-P) T P\|: P \in \operatorname{Lat} \mathscr{S}\} .
$$

In certain cases, in particular when $W$ is a dual Banach space, we have that $d_{Y}(T, \mathscr{S})$ is always $\operatorname{dist}(T, \mathscr{S})$. More generally, if the unit ball of $B(V, W)$ is compact in the weak operator topology, then it follows from Theorem 2.7(1) that $d_{Y}(T, \mathscr{S})$ is always $\operatorname{dist}(T, \mathscr{S})$. If $Y$ is the set of weak-operator continuous linear functionals, then $d_{Y}(T, \mathscr{S})$ is always the norm distance from $T^{\#}$ to the closure of $\left\{S^{\#}: S \in \mathscr{S}\right\}$ in the topology of pointwise weak *-convergence (the weak*-operator topology); this follows from the fact that the unit ball of $B\left(W^{\#}, V^{\#}\right)$ is always compact in the weak *-operator topology. Regardless of $V$ and $W$, if $Y=B(V, W)^{\#}$, then $d_{Y}(T, \mathscr{S})$ is always $\operatorname{dist}(T, \mathscr{S})$.

We will restrict ourselves to the situation in which $V$ and $W$ are normed spaces, $E$ is the set of continuous rank-one tensors on $B(V, W)$, and $Y$ is a linear subspace of $B(V, W)$ containing $E$ such that $d_{Y}(T, \mathscr{S})=\operatorname{dist}(T, \mathscr{S})$ for every $T$ in $B(V, W)$ and every $\sigma(X, Y)$-closed linear subspace $\mathscr{S}$ of $B(V, W)$. We say that a linear subspace $\mathscr{S}$ of $B(V, W)$ is hyperreflexive if and only if $\mathscr{S}$ is $E$-hyperreflexive, and we call $K(\mathscr{S})=K_{E}(\mathscr{S})$ the constant of hyperreflexivity of $\mathscr{S}$. In this case we have a distance formula for every $T$ in $B(V, W)$ :

$$
\operatorname{dist}(T, \mathscr{S}) \leq K(\mathscr{S}) d_{E}(T, \mathscr{S}) .
$$

The preceding lemma shows that this coincides with the notion of hyperreflexivity introduced by $\mathrm{W}$. Arveson in [Ar2].

As an immediate consequence of Theorem 2.2 we have that a norm closed linear subspace $\mathscr{S}$ of $B(V, W)$ is hyperreflexive with hyperreflexivity constant $K$ if and only if the set of norm continuous linear functionals with norm $1 / K$ is contained in the weak ${ }^{*}$-closed convex hull of the set of rank-one tensors of norm 1 that annihilate $\mathscr{S}$. In the case in which $W$ is the dual of a Banach space and $B(V, W)$ has a weak *-topology, Theorem 2.2 translates into the following operator-theoretic generalization of Arveson's characterization of hyperreflexivity [Ar2].

Theorem 6.7. Suppose $V$ is a Banach space and $W$ is the dual of a Banach space. $A$ weak ${ }^{*}$-closed linear subspace of $B(V, W)$ is hyperreflexive if and only if every weak*-continuous linear functional that annihilates $\mathscr{S}$ is an $l^{1}$-sum of rank-one tensors that annihilate $\mathscr{S}$.

We also obtain similar generalizations of results in [L2, KL] as an immediate interpretation of Theorem 2.3.

Theorem 6.8. Suppose $V$ is a Banach space and $W$ is the dual of a Banach space, and $\mathscr{S}$ is a hyperreflexive linear subspace of $B(V, W)$.

(1) Every weak*-closed linear subspace of $\mathscr{S}$ is hyperreflexive if and only if every weak*-continuous linear functional on $B(V, W)$ can be represented on $\mathscr{S}$ as a single rank-one tensor. 
(2) If $r \geq 1$, and every weak ${ }^{*}$-continuous linear functional $\varphi$ can be represented on $\mathscr{S}$ as a rank-one tensor with norm at most $r\|\varphi\|$, then the hyperreflexivity constant for every weak*-closed linear subspace of $\mathscr{S}$ is at most $(r+1)(K(S)+1)-1$.

(3) Suppose $\mathscr{S}$ satisfies the hypothesis of (2) and $V_{1}, W_{1}$ are Banach spaces with $W_{1}$ the dual of a Banach space. If $\pi: \mathscr{S} \rightarrow B\left(V_{1}, W_{1}\right)$ is linear and continuous with respect to the weak ${ }^{*}$-topologies, then $\operatorname{Graph}(\pi)$ is a hyperreflexive subspace of $B\left(V \oplus V_{1}, W \oplus W_{1}\right)$.

Remark. The preceding theorem remains true if all references to the weak *topology are replaced by the weak operator topology.

One interesting application of the preceding result on reflexive graphs concerns polynomially bounded operators. An element $b$ in a Banach algebra is polynomially bounded $[\mathrm{H}]$ if there is a positive constant $M$ such that, for every polynomial $p(z)$, we have

$$
\|p(b)\| \leq M \sup \{|p(z)|:|z| \leq 1\} .
$$

Equivalently, if $D$ is the closed unit disk in the plane and $\mathscr{A}$ is the set of continuous complex functions on $D$ that are analytic on the open disk, $b$ is polynomially bounded if there is a bounded unital homomorphism from $\mathscr{A}$ into the Banach algebra that sends the function $f(z)=z$ to $b$.

Let $H^{\infty}$ denote the Banach space of bounded analytic functions on the unit disk. If $T$ is a polynomially bounded operator on a Hilbert space $H$, it was shown by Mlak [M] that $T$ is similar to a direct sum $A \oplus U$ such that $U$ is a unitary operator whose spectral measure is singular with respect to linear Lebesgue measure on the unit circle and for which there is a bounded unital homomorphism $\rho: H^{\infty} \rightarrow B(H)$ that sends $f(z)=z$ to $A$ and is continuous with respect to the weak ${ }^{*}$ topologies on $H^{\infty}$ and $B(H)$. Let $S$ denote the unilateral shift operator on $l^{2}$. It was shown by K. Davidson [D2] that the unital weakly closed algebra $\mathscr{A}_{w}(S)$ generated by $S$ is hyperreflexive. It follows that $\mathscr{A}_{w}(S \oplus A \oplus U)=\mathscr{A}_{w}(S \oplus A) \oplus \mathscr{A}_{w}(U)$. Moreover, it follows that $\mathscr{A}_{w}(S \oplus A)=$ $\left\{\varphi(S) \oplus \rho(\varphi): \varphi \in H^{\infty}\right\}$, which is clearly a graph whose domain is $\mathscr{A}_{w}(S)$. These ideas lead us to the following consequence of Theorem 2.5.

Theorem 6.9. If $T$ is a polynomially bounded operator on a Hilbert space and $S$ is the unilateral shift operator, then every weak ${ }^{*}$-closed linear subspace of $\mathscr{A}_{w}(S \oplus T)$ is hyperreflexive.

We now consider direct sums as defined in $\S 4$. It follows from the definition that if a normed space $V$ is a normed direct sum of a collection $\left\{V_{\omega}: \omega \in \Omega\right\}$ of subspaces, and if $\left\{T_{\omega}: \omega \in \Omega\right\}$ is a family of operators with $T_{\omega} \in B\left(V_{\omega}\right)$ for each $\omega$ in $\Omega$, and if $\sup _{\omega}\left\|T_{\omega}\right\|<\infty$, then $T \mid V_{\omega}=T_{\omega}$ defines an operator $T$ on $V$ with norm $\sup _{\omega}\left\|T_{\omega}\right\| ; T$ is called the direct sum of the $T_{\omega}$ 's, and we write $T=\sum^{\oplus} T_{\omega}$. If $\mathscr{S}_{\omega}$ is a linear subspace of $B\left(V_{\omega}\right)$ for each $\omega$ in $\Omega$, we define $\Sigma^{\oplus} \mathscr{S}_{\omega}=\left\{\sum^{\oplus} T_{\omega}: T_{\omega} \in \mathscr{S}_{\omega}\right.$ for every $\omega$ in $\left.\Omega\right\}$.

Theorem 6.10. Suppose the Banach space $V$ is a normed direct sum of the collection $\left\{V_{\omega}: \omega \in \Omega\right\}$ of Banach spaces such that $\sum V_{\omega}$ is dense in $V$. Assume that $\left\{\mathscr{S}_{\omega}: \omega \in \Omega\right\}$ is a collection of linear spaces, $\mathscr{S}_{\omega} \subset B\left(V_{\omega}\right)$ for each $\omega$ in $\Omega$. Then

(1) $\sum^{\oplus} B\left(V_{\omega}\right)$ is hyperreflexive in $B(V)$ with $K\left(\sum^{\oplus} B\left(V_{\omega}\right)\right) \leq 4$, 
(2) $\sum^{\oplus} \mathscr{S}_{\omega}$ is a reflexive subspace of $B(V)$ if and only if each $\mathscr{S}_{\omega}$ is a reflexive subspace of $B\left(V_{\omega}\right)$, and

(3) $\sum^{\oplus} \mathscr{S}_{\omega}$ is a hyperreflexive subspace of $B(V)$ if and only if each $\mathscr{S}_{\omega}$ is a hyperreflexive subspace of $B\left(V_{\omega}\right)$, and $\sup _{\omega} K\left(\mathscr{S}_{\omega}\right)<\infty$.

Proof. (1) For each subset $I$ of $\Omega$, let $P_{I}$ denote the direct sum $\sum^{\oplus} \chi_{I}(\omega)$ and let $U_{I}=1-2 P_{I}$; if $I=\{\omega\}$ we use the notation $P_{\omega}$ and $U_{\omega}$.

Suppose $T \in B(V)$, let $E$ denote the continuous rank-one tensors in $B(V)^{\#}$, let $d=d_{E}\left(T, \sum^{\oplus} B\left(V_{\omega}\right)\right)$, and let $\operatorname{ball}(T, 4 d)$ denote the ball centered at $T$ with radius $4 d$. Note that each $P_{I}$ and $1-P_{I}$ are in $\operatorname{Lat}\left(\sum^{\oplus} B\left(V_{\omega}\right)\right)$ and, for every $\omega$ in $\Omega$, that Lemma 6.6 implies $\left\|T-U_{I} T U_{I}\right\|=\left\|T U_{I}-U_{I} T\right\|=$ $2\left\|T P_{I}-P_{I} T\right\| \leq 2\left[\left\|\left(1-P_{I}\right) T P_{I}\right\|+\left\|P_{I} T\left(1-P_{I}\right)\right\|\right] \leq 4 d$. Let $\pi(T)=\sum^{\oplus} P_{\omega} T \mid V_{\omega}$ and let $T_{I}=P_{I} \pi(T) P_{I}+\left(1-P_{I}\right) T\left(1-P_{I}\right)$ for each finite subset $I$ of $\Omega$. Also if $I$ has $n$ elements, let $\mathscr{G}_{I}$ denote the commutative group of order $2^{n}$ generated by $\left\{U_{\omega}: \omega \in I\right\}$. A standard computation shows that

$$
\frac{1}{2^{n}} \sum_{U \in \mathscr{G}_{I}} U T U=T_{I}
$$

But ball $(T, 4 d)$ is convex; thus $T_{I} \in \operatorname{ball}(T, 4 d)$. Since $\sum V_{\omega}$ is dense in $V$, it follows that $\left\{T_{I}: I \subset \Omega, I\right.$ finite $\}$ is a net in ball $(T, 4 d)$ that converges in the strong operator topology to $\pi(T)$. Hence $\pi(T) \in \operatorname{ball}(T, 4 d)$, which implies that $\operatorname{dist}\left(T, \sum^{\oplus} B\left(V_{\omega}\right)\right) \leq 4 d_{E}\left(T, \sum^{\oplus} B\left(V_{\omega}\right)\right)$. Therefore, $K\left(B\left(V_{\omega}\right)\right) \leq 4$. This proves (1). Statements (2) and (3) are consequences of Theorems 4.1 and 2.6.

Theorem 6.11. Suppose the Banach space $V$ is a normed direct sum of the collection $\left\{V_{\omega}: \omega \in \Omega\right\}$ with $V_{\omega}=U$ for each $\omega$ in $\Omega$ such that $\sum V_{\omega}$ is dense in $V$. Suppose also that $\mathscr{S}$ is a linear subspace of $B(U)$ and $\mathscr{S}_{\Omega}$ is the set of all constant elements in $\sum^{\oplus} B\left(V_{\omega}\right)$ with coordinates in $\mathscr{S}$. Then

(1) $B(U)_{\Omega}$ is hyperreflexive and $K\left(B(U)_{\Omega}\right) \leq 24$.

(2) $\mathscr{S}_{\Omega}$ is reflexive if $\mathscr{S}$ is reflexive, and 24.

(3) $\mathscr{S}_{\Omega}$ is hyperreflexive if $\mathscr{S}$ is hyperreflexive, in fact $K\left(\mathscr{S}_{\Omega}\right) \leq 25 K(\mathscr{S})+$

Proof. (1) In view of part (1) of the preceding theorem, it suffices to show that $B(U)_{\Omega}$ is relatively hyperreflexive with respect to $\sum^{\oplus} B\left(V_{\omega}\right)$. Suppose $T=$ $\sum^{\oplus} T_{\omega} \in \sum^{\oplus} B\left(V_{\omega}\right)$. It is clear that $\operatorname{dist}\left(T, B(U)_{\Omega}\right) \leq \sup _{\omega, \omega^{\prime} \in \Omega}\left\|T_{\omega}-T_{\omega^{\prime}}\right\|$. Suppose $x \in$ ball $U$ and $\alpha \in$ ball $U^{\#}$. Define $\bar{x} \in V$ by $\bar{x}(\omega)=x / 2=-\bar{x}\left(\omega^{\prime}\right)$ and $\bar{x}$ is 0 be 0 in all other coordinates. Similarly, let $\bar{\alpha}(\omega)=\bar{\alpha}\left(\omega^{\prime}\right)=$ $\alpha / 2$ and let $\bar{\alpha}$ in all other coordinates. Then $\bar{x} \in V$ and $\bar{\alpha} \in V^{\#}$, and the rank-one tensor $\bar{x} \otimes \bar{\alpha}$ is in $B(U)_{\Omega}^{\perp}$. On the other hand, $(\bar{x} \otimes \bar{\alpha})(T)=$ $\frac{1}{4} \alpha\left(\left(T_{\omega}-T_{\omega^{\prime}}\right) x\right)$. Taking the supremum over all $x$ 's and $\alpha$ 's we obtain $\left\|T_{\omega}-T_{\omega^{\prime}}\right\| \leq 4 d_{E}\left(T, B(U)_{\Omega}\right)$. It follows that

$$
\operatorname{dist}\left(T, B(U)_{\Omega}\right) \leq 4 d_{E}\left(T, B(U)_{\Omega}\right) .
$$

Therefore $\left.K_{E}\left(B(U)_{\Omega}\right), \sum^{\oplus} B\left(V_{\omega}\right)\right) \leq 4$. It follows from Theorem 2.6 and part (1) of the preceding theorem that $K_{E}\left(B(U)_{\Omega}\right) \leq 24$.

(2) This follows from (1) and Theorem 2.6.

(3) It is easily seen that $K\left(\mathscr{S}_{\Omega}, B(U)_{\Omega}\right) \leq K(\mathscr{S})$, and it follows from Theorem 2.6 that $K\left(\mathscr{S}_{\Omega}\right) \leq 25[K(\mathscr{S})+1]-1=25 K(\mathscr{S})+24$. 
Remark. Part (3) of the preceding theorem provides an affirmative answer to Question 3.15 in $[\mathrm{KL}]$ in the case in which $U$ is a Hilbert space and $V$ is the Hilbert space direct sum of copies of $U$.

If a Banach space $V$ has a sufficiently nice basis, then $V$ can be viewed as a normed direct sum of copies of the field $F$. In this case $F_{\Omega}$ is simply the scalar multiples of the identity operator, and the preceding theorem would imply that $F_{\Omega}$ is hyperreflexive. However, the results on hyperreflexive graphs allows us to prove the result for an arbitrary normed linear space.

Proposition 6.12. Suppose $V$ is a normed space. Then the set $\Lambda$ of scalar multiples of the identity operator is a hyperreflexive subspace of $B(V)$.

Proof. The result is obvious if $\operatorname{dim} V=1$. If $\operatorname{dim} V>1$, we can write $V$ as a direct sum $V=V_{1} \oplus V_{2}$ where $\operatorname{dim}\left(V_{1}\right)=1$. Let $X_{1}=B\left(V_{1}\right)=F$ and $Y_{1}=B\left(V_{1}\right)^{\#}=F$. Let $X_{2}=B\left(V_{2}\right)$, and let $Y_{2}$ be the set of all functionals in $B\left(V_{2}\right)^{\#}$ that can be written as absolutely convergent sums of rank-one tensors. Let $E_{i}$ denote the set of rank-one tensors on $B\left(V_{1}\right)$. Let $\mathscr{S}=F \subset X_{1}$. Then $\mathscr{S}$ is hyperreflexive and $Y_{1}$ is $E_{1}$-elementary of order 1 on $\mathscr{S}$. Define $\rho: \mathscr{S} \rightarrow X_{2}$ by $\rho(\lambda)=\lambda \cdot 1$. It follows from Theorem 2.5 that $\Lambda=\operatorname{graph}(\rho)$ is $E_{1} \times E_{2}$-hyperreflexive with respect to $B\left(V_{1}\right) \oplus B\left(V_{2}\right)$. On the other hand, if $P_{i}$ is the projection of $V$ onto $V_{i}$ for $i=1,2$, then, for every $T$ in $B(V)$, $\operatorname{dist}\left(T, B\left(V_{1}\right) \oplus B\left(V_{2}\right)\right) \leq\left\|P_{1} T P_{2}\right\|+\left\|P_{2} T P_{1}\right\| \leq 2 d_{E}\left(T, B\left(V_{1}\right) \oplus B\left(V_{2}\right)\right)$. Hence $B\left(V_{1}\right) \oplus B\left(V_{2}\right)$ is relatively hyperreflexive with respect to $B(V)$. Hence, by Theorem 2.6, $\Lambda$ is hyperreflexive in $B(V)$.

The proof of K. Davidson [D2] that $\mathscr{A}_{w}(S)$ is hyperreflexive can be modified to prove the same result for the unilateral shift operator on $l^{1}$. Suppose that $A$ is the unilateral shift operator on $l^{1}$ and $\left\{e_{n}: n \geq 0\right\}$ is the usual basis for $l^{1}$. Suppose that $T \in B\left(l^{1}\right)$ and $A T=T A$. If $A e_{0}=\sum_{n=0}^{\infty} a_{n} e_{n}$ with $\left\{a_{n}\right\}$ in $l^{1}$, then it follows that $\sum_{n=0}^{\infty} a_{n} A^{n}$ is absolutely convergent and agrees with $T$ on each basis vector, i.e.,

$$
T e_{m}=T A^{m} e_{0}=A^{m} T e_{0}=\sum_{n=0}^{\infty} a_{n} A^{n+m} e_{0}=\left[\sum_{n=0}^{\infty} a_{n} A^{n}\right] e_{m} .
$$

Hence $\mathscr{A}_{w}(A)=\left\{\sum_{n=0}^{\infty} a_{n} A^{n}:\left\{a_{n}\right\} \in l^{1}\right\}$. Since $\mathscr{A}_{w}(A) e_{0}=l^{1}$, it follows that $\mathscr{A}_{w}(A)$ is strictly cyclic. Moreover, the map $T \mapsto T e_{0}$ is an isometry, so it follows from [La] that every norm continuous linear functional $\varphi$ on $\mathscr{A}_{w}(A)$ can be represented by a single rank-one tensor $e_{0} \otimes \alpha$ with $\|\alpha\|=\|\varphi\|$. It follows that if $E$ is the set of rank-one tensors on $B\left(l^{1}\right)$, then $B\left(l^{1}\right)^{\#}$ is $E$ elementary on $\mathscr{A}_{w}(A)$. Since the algebra $\mathscr{A}_{w}(A)$ is isomorphic to the Banach algebra $l^{1}$ under involution, it follows that $\mathscr{A}_{w}(A)$ is semisimple. It follows from [La, Theorem 5.2] (see also [H2, Theorem 8]) that $\mathscr{A}_{w}(A)$ is reflexive. The proof of hyperreflexivity using Davidson's techniques [D2], requires the following lemma. Let $\mathscr{L}$ denote the set of all operators on $l^{1}$ having a lower triangular matrix and let $\mathscr{T}$ denote the set of all operators on $l^{1}$ whose matrices are Toeplitz matrices (i.e., constant on diagonals parallel to the main diagonal). Let $R$ denote the backwards unilateral shift on $l^{1}$. A simple computation shows that $T \in \mathscr{T}$ if and only if $R T A=T$.

Lemma 6.13. (1) $\mathscr{L}$ is hyperreflexive in $B\left(l^{1}\right)$ with $K(\mathscr{L})=1$. 
(2) There is a unital surjective contractive idempotent linear map $\pi: B\left(l^{1}\right) \rightarrow$ $\mathscr{T}$ such that

(a) $\pi(\mathscr{L}) \subset \mathscr{A}_{w}(A)$, and

(b) $\pi(T)$ is in the weak ${ }^{*}$-closed convex hull of $\left\{R^{n} T A^{n}: n \geq N\right\}$ for every $T$ in $B\left(l^{1}\right)$ and every positive integer $N$.

Note that part (2) below applies to power bounded operators on arbitrary spaces rather than just polynomially bounded operators as in Theorem 6.9.

Theorem 6.14. Suppose $V$ is a normed linear space, $T \in B(V)$ and $\sup _{n \geq 0}\left\|T^{n}\right\|$ $<\infty$. Then

(1) $\mathscr{A}_{w}(A)$ is hyperreflexive in $B\left(l^{1}\right)$, and

(2) $\mathscr{A}_{w}(A \oplus T)$ is hyperreflexive in $B\left(l^{1} \oplus V\right)$.

Proof. (1) This is obtained from the preceding lemma by imitating the proof of [D2, Lemma 8].

(2) Let $X_{1}=B\left(l^{1}\right), Y_{1}=B(V)^{\#}, X_{2}=B(V)$, and let $Y_{2}$ be the set of all functionals in $B(V)^{\#}$ that can be written as an absolutely convergent sum of rank-one tensors. Let $E_{1}$ and $E_{2}$ denote the rank-one tensors on $B\left(l^{1}\right)$ and $B(V)$, respectively. If we define $\rho: \mathscr{A}_{w}(A) \rightarrow B(V)$ by $\rho\left(\sum_{n=0}^{\infty} a_{n} A^{n}\right)=$ $\sum_{n=0}^{\infty} a_{n} T^{n}$, then, by Theorem $2.5, \operatorname{graph}(\rho)$ is hyperreflexive with respect to $B\left(l^{1}\right) \oplus B(V)$, which implies that $\operatorname{graph}(\rho)$ is weakly closed. Hence $\operatorname{graph}(\rho)=$ $\mathscr{A}_{w}(A \oplus T)$. As in the proof of Proposition 6.12, it follows that $B\left(l^{1}\right) \oplus B(V)$ is hyperreflexive in $B\left(l^{1} \oplus V\right)$, and, by Theorem 2.6, $\mathscr{A}_{w}(A \oplus T)$ is hyperreflexive.

We turn now to direct integrals. Suppose that $V$ is a separable, classically reflexive (i.e., $V=V^{\# \#)}$ Banach space. Let $X=B(V)$, let $E$ be the set of rankone tensors on $B(V)$, and let $Y$ denote the set of weak-operator continuous linear functionals on $B(V)$. Since $V$ and $V^{\#}$ are both separable, ball $(X)$ is a complete separable metric space in the $\sigma(X, Y)$ (weak operator) topology. Suppose that $(\Omega, \mu)$ is a probability measure space, $1<p<\infty$, and $1 / p+$ $1 / q=1$. We write $\mathscr{V}=L^{p}(\mu, V)=\int_{\Omega, p}^{\oplus} V d \mu(\omega)$ with the $p$-norm. It is known that the dual of $\mathscr{V}$ is $\mathscr{V}^{\#}=L^{q}\left(\mu, V^{\#}\right)$ since the reflexive Banach space $V$ has the Radon-Nikodym property [DU]. It follows that $\mathscr{V}$ is also a reflexive Banach space. In the standard theory of direct integrals [Di], $V$ is a separable Hilbert space and $p=2$.

Let $\mathscr{X}=L_{Y}^{\infty}(\mu, X)$ with the essential-supremum norm and let $\mathscr{Y}=$ $L_{X}^{1}(\mu, Y)$. there is a natural identification of $\mathscr{X}$ as a subalgebra of $B(\mathscr{V})$ defined, for $\varphi \in \mathscr{Z}$ and $f \in \mathscr{V}$, by $(\varphi f)(\omega)=\varphi(\omega) f(\omega)$. It is clear that the operator norm of $\varphi$ and the essential supremum norm coincide. In the standard theory of direct integrals [Di] the operators in $\mathscr{Z}$ are called decomposable, and the common notation for the operator associated with $\varphi$ is $\int_{\Omega}^{\oplus} \varphi(\omega) d \mu(\omega)$.

The $\sigma(\mathscr{Z}, \mathscr{Y})$-topology on $\mathscr{X}$ contains the weak operator topology on $\mathscr{X}$. To see this, note that if $f \in L_{V^{*}}^{p}(\mu, V)$ and $g \in L_{V}^{q}\left(\mu, V^{\#}\right)$, then $\alpha(\omega)=$ $f(\omega) \otimes g(\omega)$ defines an element of $\mathscr{Y}$ and

$$
\langle\varphi, \alpha\rangle=\langle\varphi, f \otimes g\rangle=\int_{\Omega}\langle\varphi(\omega) f(\omega), g(\omega)\rangle d \mu(\omega)
$$

for every $\varphi$ in $\mathscr{X}$. Thus the set $\mathscr{E}$ of rank-one tensors coincides with $L_{X}^{1}(\mu, E)$. Thus reflexivity and hyperreflexivity in $B(\mathscr{V})$ are $\mathscr{E}$-reflexivity and $\mathscr{E}$-hyperreflexivity, respectively. 
In addition, it is possible to identify $L^{\infty}(\mu)$ with the linear subspace $\mathscr{D}$ of $\mathscr{X}$ consisting of the functions $\varphi$ such that $\varphi(\omega)$ is a scalar multiple of the identity a.e. $(\mu)$. It is clear that the identification is isometric and is continuous with respect to the weak ${ }^{*}$ topology on $L^{\infty}(\mu)$ and the weak operator topology on $B\left(L_{V^{*}}^{p}(\mu, V)\right)$. In the standard theory of direct integrals, the operators associated with $\varphi$ 's in $L^{\infty}(\mu)$ are called scalar operators.

The first two parts of the following lemma are generalizations of the wellknown fact in the standard theory of direct integrals that the sets of decomposable operators and scalar operators are commutants of each other.

Proposition 6.15. The following are true.

(1) $\mathscr{X}$ is the commutant of $\mathscr{D}$ in $B(\mathscr{V})$.

(2) $\mathscr{D}$ is the commutant of $\mathscr{X}$ in $B(\mathscr{V})$.

(3) $\mathscr{X}$ is a hyperreflexive subalgebra of $B(\mathscr{V})$ and $K(\mathscr{X}) \leq 4$.

Proof. (1) Suppose $T \in B(\mathscr{V})$ and $T$ commutes with every operator in $\mathscr{D}$. Since $V$ is separable, we can choose a countable dense subset $V_{0}$ of $V$ that is closed under addition and under multiplication by the field $\mathscr{F}$ of scalars with rational real and imaginary parts. For each $x$ in $V_{0}$, let $\bar{x}$ denote the element of $\mathscr{V}$ defined by $\bar{x}(\omega)=x$. For each $x$ in $V_{0}$ we choose a function $\hat{x}$ in the equivalence class of $T \bar{x}$. The linearity of $T$ implies, for almost every $\omega$ in $\Omega$, that the map $x \mapsto \hat{x}(\omega)$ is $\mathscr{F}$-linear. We now want to show that, for each $x$ in $V_{0}$ and for almost every $\omega$ in $\Omega$, we have

$$
\|\hat{x}(\omega)\| \leq\|T\|\|x\| \text {. }
$$

To see this, suppose $\varepsilon>0$ and let $A=\left\{\omega \in \Omega:\|\hat{x}(\omega)\|^{p} \geq\|T\|^{p}\|x\|^{p}+\varepsilon\right\}$. Since $\chi_{A} \in \mathscr{D}$, we have $T \chi_{A} \bar{x}=\chi_{A} T \bar{x}$. Hence $\left\|T \chi_{A} \bar{x}\right\|^{p}=\int_{A}\|\hat{x}(\omega)\|^{p} d \mu(\omega)$ $\geq \int_{A}\|T\|^{p}\|x\|^{p}+\varepsilon d \mu(\omega) \geq\|T\|^{p}\left\|\chi_{A} \bar{x}\right\|^{p}+\varepsilon \mu(A)$; whence $\mu(A)=0$.

It follows from $(*)$ above that, for almost all $\omega$ in $\Omega$, the map $x \mapsto \hat{x}(\omega)$ extends uniquely to an operator $T_{\omega}$ in $B(V)$ with $\left\|T_{\omega}\right\| \leq\|T\|$.

To show that the map $\omega \mapsto T_{\omega}$ is measurable with respect to the strong operator topology, it suffices to show, for each $x$ in $V$, that the map $\omega \mapsto T_{\omega} x$ is measurable from $\Omega$ into $V$. However, the latter map coincides with $\hat{x}$ for every $x$ in $V_{0}$, and since the limit of a sequence of pointwise a.e. convergent measurable maps is measurable, we conclude that the map $\varphi: \omega \mapsto T_{\omega}$ is in $\mathscr{X}$. The operator $T-\varphi$ annihilates all vectors of the form $\chi_{A} \bar{x}$ with $A$ measurable and $x$ in $V_{0}$; since the closed linear spans of these vectors is $\mathscr{V}$, we have $T=\varphi$. Thus $T \in \mathscr{Z}$.

(2) Suppose $T$ is in the commutant of $\mathscr{X}$. Since $\mathscr{D} \subset \mathscr{X}$, it follows from (1) that $T \in \mathscr{X}$. Since $V$ and $V^{\#}$ are norm separable, the unit ball of $B(V)$ is separable and metrizable in the strong operator topology and hence contains a countable dense subset $\mathscr{B}$. For each $B$ in $\mathscr{B}$, define $\bar{B}$ in $\mathscr{X}$ by $\bar{B}(\omega)=B$. Hence $(T \bar{B})(\omega)=T(\omega) B$ a.e. $(\mu)$ and $(\bar{B} T)(\omega)=B T(\omega)$ a.e. $(\mu)$. Hence, for almost every $\omega, T(\omega)$ commutes with every element of $\mathscr{B}$, which implies $T(\omega)$ is a scalar a.e. $(\mu)$. This means $T \in \mathscr{D}$.

(3) The reflexivity of $\mathscr{X}$ follows from the fact that $\mathscr{X}$ is the commutant of the set $\mathscr{P}$ of projections in $\mathscr{D}$, since the norm closed linear span of $\mathscr{P}$ is $\mathscr{D}$. The proof that $K(\mathscr{Z}) \leq 4$ can be obtained via a direct translation of the proof of [Ro, Theorem 2.1] using the amenability of the group $\{1-2 P: P \in \mathscr{D}, P=$ $\left.P^{2}\right\}$ (see also the proof of Theorem 6.4(1)). 
Since $\mathscr{X}$ is a hyperreflexive subspace of $B(\mathscr{V})$, we can use Theorem 2.6 to show that a subspace of $\mathscr{X}$ is reflexive (hyperreflexive) in $B(\mathscr{V})$ if and only if it is relatively $\mathscr{E}$-reflexive $(\mathscr{E}$-hyperreflexive) in $\mathscr{Z}$. We may therefore directly apply all of the results of $\S 3$. Note that while the theory of direct integrals we have presented here is much more general than the standard Hilbert space version, it is still only a very special case of the version in $\S 3$.

Suppose $\left\{\mathscr{S}_{\omega}: \omega \in \Omega\right\}$ is a measurable family of $\sigma(X, Y)$-closed linear subspaces of $X$. We let $\int_{\Omega}^{\oplus} \mathscr{S}_{\omega} d \mu(\omega)$ denote $\int_{\Omega, \mathscr{Z}}^{\oplus} \mathscr{S}_{\omega} d \mu(\omega)$. Here is a translation of the major results in $\S 3$. Note that part (3) of the following theorem is new even in the standard version of direct integrals in which $V$ is a Hilbert space and $p=2$.

Theorem 6.16. If $\left\{\mathscr{S}_{\omega}: \omega \in \Omega\right\}$ is a measurable family of weak-operator-closed linear subspaces of $B(V)$, and $\mathscr{X}, \mathscr{Y}, p, q$ are as above, then

(1) if $Y$ is E-elementary of order $r$ on almost every $\mathscr{S}_{\omega}$, then $\mathscr{Y}$ is $\mathscr{E}$ elementary of order $r$ on $\int_{\Omega}^{\oplus} \mathscr{S}_{\omega} d \mu(\omega)$.

(2) $\int_{\Omega}^{\oplus} \mathscr{S}_{\omega} d \mu(\omega)$ is reflexive if and only if $\mathscr{S}_{\omega}$ is reflexive a.e. $(\mu)$.

(3) If $Y$ is E-elementary of order $r$ on almost every $\mathscr{S}_{\omega}$, and if $\int_{\Omega}^{\oplus} \mathscr{S}_{\omega} d \mu(\omega)$ is reflexive (resp. hyperreflexive), then so is every weak-operator closed linear subspace of $\int_{\Omega}^{\oplus} \mathscr{S}_{\omega} d \mu(\omega)$.

(4) $\int_{\Omega}^{\oplus} \mathscr{S}_{\omega} d \mu(\omega)$ is hyperreflexive precisely when almost every $\mathscr{S}_{\omega}$ is hyperreflexive and ess-sup $K\left(\mathscr{S}_{\omega}\right)<\infty$.

Corollary 6.17. Every weakly closed subalgebra of $\mathscr{D}$ is hyperreflexive in $B(\mathscr{V})$. Proof. Let $\mathscr{S}_{\omega}$ denote the set of scalars in $B(V)$ for each $\omega$ in $\Omega$. Then $\mathscr{D}=\int_{\Omega}^{\oplus} \mathscr{S}_{\omega} d \mu(\omega)$. It follows from Proposition 6.12 that $\sup _{\omega} K\left(\mathscr{S}_{\omega}\right)<\infty$; whence, by part (4) of the preceding theorem, $\mathscr{D}$ is hyperreflexive. The proof is completed by applying part (3) of the preceding theorem.

We next provide applications of part (4) of the preceding theorem. A quasinormal operator $T$ on a Hilbert space $H$ satisfies $T\left(T^{*} T\right)=\left(T^{*} T\right) T$. On a separable Hilbert space every quasinormal operator is a direct integral of operators that are either scalar multiples of either the identity or the unilateral shift operator (see [E]). (The hyperreflexivity of the algebra generated by the shift is proved in [D2].)

Corollary 6.18. The weak-operator closed unital algebra generated by a quasinormal operator on a Hilbert space is hyperreflexive.

Proof. The weak-operator closed algebra generated by (a scalar multiple of) the unilateral shift operator or the identity operator have the required properties to apply part (4) of the preceding theorem.

\section{APPROXIMATE REFLEXIVITY}

Suppose $V$ and $W$ are normed linear spaces, let $X=B(V, W)$, let $Y=$ $B(V, W)^{\#}$, and let $\mathscr{E}$ be the set of functionals on $X$ that are bounded limits of rank-one tensors. More precisely, $\varphi \in \mathscr{E}$ if and only if there are bounded nets $\left\{e_{\lambda}\right\}$ in $V$ and $\left\{\beta_{\lambda}\right\}$ in $W^{\#}$ such that, for every $T$ in $B(V, W)$, we 
have $\varphi(T)=\lim _{\lambda}\left\langle T e_{\lambda}, \beta_{\lambda}\right\rangle$. If $\mathscr{S}$ is a linear subspace of $B(V, W)$, we use Appr $\operatorname{Ref} \mathscr{S}$ to denote $\operatorname{Ref}_{\mathscr{E}}(\mathscr{S})$, and we say that $\mathscr{S}$ is approximately reflexive if $\mathscr{S}$ is $\mathscr{E}$-reflexive. As in the case of $\operatorname{Ref} \mathscr{S}$, there are several characterizations of Appr $\operatorname{Ref} \mathscr{S}$. A comparison of the following lemma with Lemma 6.1 shows why the term approximate reflexivity is used. The proof is elementary and is left to the interested reader.

Lemma 7.1. Suppose $\mathscr{S}$ is a linear subspace of $B(V, W)$ and $T \in B(V, W)$. The following are equivalent:

(1) $T \in \operatorname{Appr} \operatorname{Ref} \mathscr{S}$

(2) $\left\|A_{\lambda} T B_{\lambda}\right\| \rightarrow 0$ for all bounded nets $\left\{A_{\lambda}\right\}$ in $B(W)$ and $\left\{B_{\lambda}\right\}$ in $B(V)$ such that, for each $S$ in $\mathscr{S},\left\|A_{\lambda} S B_{\lambda}\right\| \rightarrow 0$,

(3) $\left\|P_{\lambda} T Q_{\lambda}\right\| \rightarrow 0$ for all nets $\left\{P_{\lambda}\right\}$ of idempotents in $B(W)$ and $\left\{Q_{\lambda}\right\}$ of idempotents in $B(V)$ such that $\lim _{\lambda}\left\|P_{\lambda}\right\|=\lim _{\lambda}\left\|Q_{\lambda}\right\|=1$, and, for each $S$ in $\mathscr{S},\left\|P_{\lambda} S Q_{\lambda}\right\| \rightarrow 0$.

As in the algebraic case, the smallest possible choice for $Y$ is $\operatorname{sp} \mathscr{E}$. The $\sigma(X, \mathrm{sp} \mathscr{E})$-topology is called the approximate weak-operator topology, abbreviated as the a.w. topology. One other possible topology on $B(V, W)$ is the weak topology, i.e., the $\sigma\left(B(V, W), B(V, W)^{\#}\right)$-topology. These topologies generally do not coincide; a weak-operator continuous linear functional is in $\mathscr{E}$ if and only if it is a rank-one tensor.

There are two versions of the Loginov-Shulman theorem for approximate reflexivity.

Theorem 7.2. Suppose $\mathscr{S}$ is an approximately reflexive linear subspace of $B(V, W)$. Then

(1) every a.w. closed linear subspace of $\mathscr{S}$ is approximately reflexive if and only if every a.w. continuous linear functional on $B(V, W)$ can be represented on $\mathscr{S}$ by an element of $\mathscr{E}$.

(2) Every norm closed linear subspace of $\mathscr{S}$ is approximately reflexive if and only if each norm continuous linear functional on $B(V, W)$ can be represented on $\mathscr{S}$ by an element of $\mathscr{E}$.

Remark. It may seem unlikely that a linear subspace $\mathscr{S}$ of $B(V, W)$ has the property that every norm continuous linear functional in $B(V, W)^{\#}$ can be represented on $\mathscr{S}$ as an element of $\mathscr{E}$. However, if $E$ is the set of all rankone tensors, and $\operatorname{sp} E$ is $E$-elementary of order $r$ on $\mathscr{S}$, then $B(V, W)^{\#}$ is $\mathscr{E}$-elementary of order $r$ on $\mathscr{S}$. To see this, suppose $\varepsilon>0$ and $\varphi \in B(V, W)^{\#}$ and $\|\varphi\|=1$. Then there is a net $\left\{\varphi_{\lambda}\right\}$ of unit vectors in $\operatorname{sp} E$ such that $\varphi_{\lambda} \rightarrow \varphi$ $\left(\right.$ weak $\left.^{*}\right)$. It follows from the hypothesis that there is a net $\left\{e_{\lambda}\right\}$ in $E$ such that, for each $\lambda$, we have $\varphi_{\lambda}-e_{\lambda} \in \mathscr{S}^{\perp}$ and $\left\|e_{\lambda}\right\| \leq(r+\varepsilon)\left\|\varphi_{\lambda}\right\| \leq r+\varepsilon$. There is a weak $^{*}$-convergent subnet of $\left\{e_{\lambda}\right\}$ with limit $e \in \mathscr{E}$ such that $\varphi-e \in \mathscr{S}^{\perp}$ and $\|e\| \leq r+\varepsilon$.

Other results in the preceding section have natural analogues in the approximate setting. If $V_{1}$ and $V_{2}$ are normed vector spaces, we always give $V_{1} \oplus V_{2}$ the product topology.

Proposition 7.3. Suppose $\mathscr{S}$ is a linear subspace of $B(V, W)$. Then

(1) if $n$ is a positive integer, then $\operatorname{Appr} \operatorname{Ref}\left(\mathscr{S}^{(n)}\right)=\left[\operatorname{Ref}_{\mathscr{E}_{n}}(\mathscr{S})\right]^{(n)}$. In particular, $\mathscr{S}^{(n)}$ is approximately reflexive if and only if $\mathscr{S}$ is $E_{n}$-reflexive. 
(2) A transformation $T$ in $B(V, W)$ is in the a.w. closure of $\mathscr{S}$ if and only if, for each positive integer $n, T^{(n)} \in \operatorname{Appr} \operatorname{Ref} \mathscr{S}^{(n)}$.

(3) If $\mathscr{S}$ is a.w. closed and every a.w. continuous linear functional can be represented on $\mathscr{S}$ as an element of $\mathscr{E}$, then $\mathscr{S}^{(3)}$ is approximately reflexive.

The results of the preceding section on reflexive graphs carry over directly in the approximate setting. Suppose that $V_{1}, V_{2}, W_{1}$, and $W_{2}$ are normed spaces, $V=V_{1} \oplus V_{2}$ and $W=W_{1} \oplus W_{2}$. It is easily seen that $B\left(V_{1}, W_{1}\right) \oplus B\left(V_{2}, W_{2}\right)$ is an approximately reflexive subalgebra of $B(V, W)$ (since it is reflexive).

Proposition 7.4. Suppose that $\mathscr{S}$ is an approximately reflexive subspace of $B\left(V_{1}, W_{1}\right)$ and $\rho: \mathscr{S} \rightarrow B\left(V_{2}, W_{2}\right)$ is a norm continuous linear map. The following are true.

(1) If every a.w. closed linear subspace of $\mathscr{S}$ is reflexive and if $\rho$ is continuous with respect to the a.w. topologies on $\mathscr{S}$ and $B\left(V_{2}, W_{2}\right)$, then $\operatorname{graph}(\rho)$ is an approximately reflexive linear subspace of $B\left(V_{1} \oplus V_{2}, W_{1} \oplus W_{2}\right)$.

(2) If every norm closed linear subspace of $\mathscr{S}$ is approximately reflexive, then $\operatorname{graph}(\rho)$ is approximately reflexive.

There is another version of approximate reflexivity that has been studied. Suppose $V=W$ and $\mathscr{A}$ is a unital subalgebra of $B(V)$. If $M$ is a closed linear subspace of $V$, let $\pi_{M}$ denote the quotient map $\pi_{M}: V \rightarrow V / M$. We define Appr Alg Lat $\mathscr{A}$ to be the set of operators $T$ for which $\left\|\left.\pi_{M_{\lambda}} T\right|_{M}\right\| \rightarrow 0$ for every net $\left\{M_{\lambda}\right\}$ of closed subspaces of $V$ such that, for every $A$ in $\mathscr{A},\left\|\left.\pi_{M_{\lambda}} A\right|_{M}\right\| \rightarrow 0$. If $V$ is a Hilbert space and $P$ is the projection onto $M$, then $\left\|\left.\pi_{M} T\right|_{M}\right\|=\|(1-P) T P\|$. This notion of approximate reflexivity was introduced in [AFV1, AFV2, and H1]. In [H1] it was shown that Appr Alg Lat $\mathscr{A}=\mathscr{A}$ whenever $\mathscr{A}$ is a unital $C^{*}$-algebra of operators on a Hilbert space. In [HO] it was shown that if $K$ is a compact Hausdorff space, $V$ is a Banach space, and $m: C(K) \rightarrow B(V)$ is a bounded unital homomorphism, then $m(C(K))=$ Appr Alg Lat $m(C(K))$.

Unlike in the algebraic and topological settings, we do not know if the two notions of approximate reflexivity coincide. The following question has been recently answered in the affirmative in the case in which $V$ is a Hilbert space [H3].

Question. If $\mathscr{A}$ is a unital subalgebra of $B(V)$, must it be true that Appr Ref $\mathscr{A}$ $=$ Appr Alg Lat $\mathscr{A}$ ? If $\mathscr{A}=$ Appr Ref $\mathscr{A}$, then must $\mathscr{A}=$ Appr Alg Lat $\mathscr{A}$ ?

It is easily seen that $\mathscr{A} \subset$ Appr Ref $\mathscr{A} \subset$ Appr Alg Lat $\mathscr{A}$ always holds. Thus if $\mathscr{A}=$ Appr Alg Lat $\mathscr{A}$, then $\mathscr{A}=$ Appr Ref $\mathscr{A} ;$ it is the reverse implication that is in question. This difficulty means that we cannot apply the preceding theorems of this section to Appr Alg Lat. For example, suppose $\mathscr{A}=$ Appr Alg Lat $\mathscr{A}$ and every norm continuous linear functional can be represented on $\mathscr{A}$ by an element of $\mathscr{E}$. It is not known whether this implies that $\mathscr{B}=$ Appr Alg Lat $\mathscr{B}$ for every norm closed unital subalgebra $\mathscr{B}$ of $\mathscr{A}$.

It was proved in [AFV1, AFV2] that if $H$ is a separable Hilbert space and $\mathscr{A}$ is a norm separable unital subalgebra of $B(H)$, then Appr Alg Lat $\mathscr{A} \subset$ $[\mathscr{A}+\mathscr{K}(H)]^{-}$. We can show that this is true without the separability assumption on $\mathscr{A}$.

Proposition 7.5. If $\mathscr{A}$ is a unital algebra of operators on an arbitrary Hilbert space $H$, then Appr Alg Lat $\mathscr{A} \subset[\mathscr{A}+\mathscr{K}(H)]^{-}$. 
Proof. Let $\mathscr{C}(H)=B(H) / \mathscr{K}(H)$ denote the Calkin algebra and let $\eta: B(H) \rightarrow$ $\mathscr{C}(H)$ be the quotient map. If $\mathscr{S}$ is a subset of $\mathscr{C}(H)$, we can define Alg Lat $\mathscr{S}$ and Appr Alg Lat $\mathscr{S}$ using projections in $\mathscr{C}(H)$. It was shown by Voiculescu [V] that $\mathscr{S}=\operatorname{Alg}$ Lat $\mathscr{S}$ for every norm separable unital closed subalgebra of $\mathscr{C}(H)$ when $H$ is separable, and $\mathrm{W}$. Arveson [Arl] showed, in this case, that, given $t \in \mathscr{C}(H)$, there is a projection $p$ in $\mathscr{C}(H)$ such that $(1-p) \mathscr{S} p=\{0\}$ and $\|(1-p) t p\|=\operatorname{dist}(t, \mathscr{S})$. In [H1] both of these latter results were extended to the case in which $H$ is not separable. However, Davidson [D1] has shown that the separability assumption on $\mathscr{S}$ cannot be dropped. However, the distance estimate given in Arveson's result implies that Appr Alg Lat $\mathscr{S}=\mathscr{S}$ for every Hilbert space $H$ and every unital norm closed subalgebra $\mathscr{S}$ of $\mathscr{C}(H)$. The proof of our proposition in [AFV1] when $\mathscr{A}$ is separable involves showing that $\eta($ Appr Alg Lat $\mathscr{A}) \subset$ Alg Lat $\eta(\mathscr{A})=\eta(\mathscr{A})^{-}$. When $\mathscr{A}$ is not separable, Alg Lat $\eta(\mathscr{A})=\eta(\mathscr{A})^{-}$may no longer be true. However, [H1, Theorem 8.2] implies Appr Alg Lat $\mathscr{A}$ is the union of Appr Alg Lat $\mathscr{B}$, where $\mathscr{B}$ ranges over the separable unital closed subalgebras of $\mathscr{A}$. Since $\eta($ Appr Alg Lat $\mathscr{B})=\eta(\mathscr{B})^{-} \subset \eta(\mathscr{A})^{-}$for each $\mathscr{B}$, the proof is complete.

If we replace Appr Alg Lat with Appr Ref, we can give a much simpler proof of the preceding proposition. The key idea is a fact that follows from a theorem of J. Glimm [G] characterizing states on $B(H)$ that annihilate $\mathscr{K}(H)$, namely, if $\varphi: B(H) \rightarrow \mathbb{C}$ is a norm continuous linear functional and $\mathscr{K}(H) \subset$ $\operatorname{ker} \varphi$, then there are bounded nets $\left\{e_{\lambda}\right\}$ and $\left\{f_{\lambda}\right\}$ in $H$ such that, for each $T$ in $B(H)$, we have $\varphi(T)=\lim _{\lambda}\left(T e_{\lambda}, f_{\lambda}\right)$.

Propositon 7.6. If $\mathscr{S}$ is a linear space of operators on a Hilbert space $H$, then Appr $\operatorname{Ref} \mathscr{S} \subset[\mathscr{S}+\mathscr{K}(H)]^{-}$.

Proof. Suppose $T \in \operatorname{Appr} \operatorname{Ref} \mathscr{S}$ and let $\varphi$ be a continuous linear functional on $B(H)$ with norm 1 such that $\varphi(T)$ is the distance from $T$ to $[\mathscr{S}+\mathscr{K}(H)]^{-}$. It follows from the remarks preceding this proposition that $\varphi \in \mathscr{E}$. Hence $\varphi(T)=0$, which implies $T \in[\mathscr{S}+\mathscr{K}(H)]^{-}$.

In [Gi] Gillespie proved that if $K$ is a compact Hausdorff space, $V$ is a Banach space and $m: C(K) \rightarrow B(V)$ is a bounded unital homomorphism with weakly compact action (i.e., the map $f \rightarrow m(f) v$ is weakly compact for every $v$ in $V$ ), then there is an $r>0$ such that every weak-operator continuous linear functional with norm 1 can be represented on $m(C(K))$ by a rank-one tensor with norm at most $r$. (In fact, any $r>\|m\|$ will work.) Note that the map $m$ has weakly compact action whenever $V$ does not contain a copy of $c_{0}$ [Pel]. Gillespie's result has been recently proved without the assumption of weakly compact action [AAK]. It follows from the remark after Theorem 7.2 that every norm continuous linear functional can be represented on $m(C(K))$ by an element of $\mathscr{E}$ with norm at most $r$. Combining these ideas with the aforementioned result of [HO], we are led to the following result.

Proposition 7.7. Suppose $K$ is a compact Hausdorff space, $V$ is a Banach space and $m: C(K) \rightarrow B(V)$ is a bounded unital homomorphism. Then every norm closed linear subspace $\mathscr{S}$ of $m(C(K))$ is approximately reflexive, i.e., $\mathscr{S}=$ Appr $\operatorname{Ref} \mathscr{S}$.

Remark. It is natural to wonder if, in the above proposition, one can conclude that $\mathscr{A}=$ Appr Alg Lat $\mathscr{A}$ for every norm closed unital subalgebra of $m(C(K))$. 


\section{MiscellaNY}

1. There are various other versions of reflexivity that are not included as special cases of our general version. In [AS1 and AS2] E. Azoff and H. Shaheda have studied a version of reflexivity for convex sets rather than subspaces. This study has taken place in the setting of real or complex Banach spaces, and includes a version of the Loginov-Shulman theorem.

2. In [DF and $\mathrm{HO}$ ] a version of reflexivity (called para-reflexivity) for operator algebras on Hilbert space have been studied where the lattice of invariant subspaces has been replaced by the collection of invariant operator ranges. An operator range is the range of an operator on the same Hilbert space. As of yet, there is no analogue of para-reflexivity for linear subspaces of operators.

3. There are many notions of reflexivity based on the characterizations of $\operatorname{Ref} \mathscr{S}$ and $\operatorname{Ref}_{0} \mathscr{S}$. We can define an arbitrary subset $\mathscr{S}$ of $B(V, W)$ to be reflexive if $T \in \mathscr{S}$ whenever $T$ is an operator such that, for every vector $x \in V$, we have $T x \in(\mathscr{S} x)^{-}$. If $V$ and $W$ are vector spaces over a field, we define $\mathscr{S} \subset \mathscr{L}(V, W)$ to be algebraically reflexive if $T \in \mathscr{S}$ whenever $T \in \mathscr{L}(V, W)$ and, for every $x$ in $V$, we have $T x \in \mathscr{S} x$. If $V=W$ is a Hilbert space, $T \in B(V)$ and $\mathscr{S}$ is the strong operator closure of $\left\{T^{n}: n \geq 0\right\}$, we say that $T$ is orbit-reflexive if $\mathscr{S}$ is reflexive. Orbit-reflexivity was introduced and studied in [HNRR]. Although Read [Rd] has provided an example of an operator $T$ on a Banach space such that, for every nonzero vector $x$, $\left\{T^{n} x: \geq 0\right\}^{-}=X$, on a Hilbert space there is no known example of an operator that is not orbit-reflexive.

4. In the paper of D. Larson [L3] several notions of reflexivity were introduced. Each of these is a special case of our general notion of reflexivity, varying the choices of $X, Y$, and $E$.

5. In [H3] a notion of approximate hyperreflexivity is studied in the Hilbert space setting. It is proved in [H3] that every $C^{*}$-algebra of operators is approximately hyperreflexive. In [H4] the approximate reflexivity and approximate hyperreflexivity of the space of compact operators on a Banach space is related to the geometry of the space.

6. Suppose $(X, Y, E)$ is a reflexivity triple over a Hausdorff field $F$. We say that a linear subspace $\mathscr{S}$ is $E$-transitive if $\operatorname{Ref}_{E}(\mathscr{S})=X$, equivalently, if $E \cap$ $\mathscr{S}^{\perp}=\{0\}$. This concept generalizes both the notions of strict transitivity in the algebraic setting and the topological setting. Perhaps some general transitivity result could be proved in the general setting that would include some of the known operator-theoretic transitivity results.

7. In the case of a Hilbert space $H$, we have $B(H)=\mathscr{C}_{1}(H)^{\#}$, where $\mathscr{C}_{1}(H)$ is the set of trace class operators on $H$. The set of extreme points of the unit ball of $\mathscr{C}_{1}(H)$ is precisely the set of rank-one tensors with norm 1 . Also the extreme points of $B(H)^{\#}$ are precisely the functionals of norm 1 that are weak *limits of bounded nets of rank-one tensors. This leads naturally to a general construction of reflexivity triples that includes both reflexivity and approximate reflexivity on Hilbert space as special cases. Suppose $Y$ is a Banach space whose unit ball is the weakly closed convex hull of its set $E_{0}$ of extreme points. Let $X$ be a closed subspace of $Y^{\#}$ that separates the points of $Y$, and let $E$ be the set of all scalar multiples of the elements of $E_{0}$. Perhaps studying reflexivity triples of the form $(X, Y, E)$ thus defined can shed light on the 
Hilbert space situation. If $Y=l^{1}$ and $X=c_{0}$ or $l^{\infty}$, then the only reflexive subspaces are the ones that vanish on some subset of $\{0,1,2, \ldots\}$. On the other hand, if $Y=l^{\infty}$ and $X=l^{1}$, the situation is much more complicated (and interesting). What happens when $H$ is a Hilbert space, $X=\mathscr{C}_{1}(H)$ (the trace class operators), and $Y=B(H)=\mathscr{C}_{1}(H)^{\#}$ ? In this case $E$ is the set of all operators $T$ for which either $T^{*} T$ or $T T^{*}$ is a positive scalar multiple of the identity operator. For example, it follows from Theorem 2.1 that if $\mathscr{A}$ is a von Neumann algebra in $B(H)$, then $\mathscr{S}=\mathscr{A}_{\perp}$ is an $E$-hyperreflexive subspace of $\mathscr{C}_{1}(H)$ with $K_{E}\left(\mathscr{A}_{\perp}\right)=1$, since ball $\left(\mathscr{S}^{\perp}\right)=\operatorname{ball}(\mathscr{A})$ is the (norm) closed convex hull of the unitary operators in $\mathscr{A}$, i.e. ball $\left(\mathscr{S}^{\perp}\right) \subset \overline{\operatorname{co}}\left(\mathscr{S}^{\perp} \cap \widetilde{E}\right)$.

8. It seems possible that our intersection theorem (Theorem 2.8) might be used to show that every von Neumann algebra is hyperreflexive. Suppose that $\mathscr{A}$ is a von Neumann algebra whose commutant is generated (as a von Neumann algebra) by a single operator $T$. Then $\mathscr{A}=\{\operatorname{Re} T\}^{\prime} \cap\{\operatorname{Im} T\}^{\prime}$. However, $\{\operatorname{Re} T\}^{\prime}$ and $\{\operatorname{Im} T\}^{\prime}$ are type I von Neumann algebras and are therefore hyperreflexive (see [Ros]). Is it possible to choose $T$ so that $\{\operatorname{Re} T\}^{\prime}+\{\operatorname{Im} T\}^{\prime}$ is norm closed. Note, for example that the scalars is the intersection of the algebra $H^{\infty}$ of analytic Toeplitz operators and the algebra $\mathscr{D}$ of diagonal operators and $H^{\infty}+\mathscr{D}$ is closed. Thus one can leave the realm of selfadjoint algebras (or even algebras) to prove that a von Neumann algebra is hyperreflexive.

\section{ACKNOWLEDGMENTS}

The author discovered most of the results in the first two sections of this paper while visiting Indiana University in 1986, and he wishes to thank John Conway for providing the opportunity to visit. The author also wishes to express his gratitude to the National Science Foundation for its continued support while this research was undertaken. Many thanks are given to Ileana Ionaşcu, who read the manuscript several times and made many useful comments.

\section{REFERENCES}

[AAK] Y. A. Abramovitch, E. L. Arenson, and A. K. Kitover, Banach $C(K)$-modules and operators preserving disjointness, Pitman Research Notes, no. 277, Wiley, New York, 1992.

[AFV1] C. Apostol, C. Foiaş, and D. Voiculescu, Strongly reductive operators are normal, Acta Sci. Math. (Szeged) 38 (1976), 261-263.

[AFV2] __, On strongly reductive algebras, Rev. Roumaine Math. Pures Appl. 21 (1976), 633642.

[Ar1] W. B. Arveson, Notes on extensions of $C^{*}$-algebras, Duke Math. J. 44 (1977), 329-355.

[Ar2] _ Ten lectures on operator algebras, CBMS Regional Conf. Ser. in Math., no. 55, Amer. Math. Soc., Providence, RI, 1984.

[Ar3] _ An invitation to $C^{*}$-algebras, Springer-Verlag, New York, 1976.

[Az] E. A. Azoff, On finite rank operators and preannihilators, Mem. Amer. Math. Soc., No. 357, 1986.

[AFG] E. Azoff, C.-K. Fong, and F. Gilfeather, A reduction theory for non-self-adjoint operator algebras, Trans. Amer. Math. Soc. 224 (1976), 351-366.

[AS1] E. A. Azoff and H. Shaheda, On separation by families of linear functionals, J. Funct. Anal. 96 (1991), 96-116.

[AS2] _ From algebras of normal operators to intersecting hyperplanes, Proc. Sympos. Pure. Math., vol. 51, Amer. Math. Soc., Providence, RI, 1990, pp. 11-16. 
[D1] K. Davidson, On operators commuting with Toeplitz operators modulo the compact operators, J. Funct. Anal. 24 (1977), 291-302.

[D2] _ The distance to the analytic Toeplitz operators, Illinois J. Math. 31 (1987), 265-273.

[DeFi] J. A. Deddens and P. A. Fillmore, Reflexive linear transformations, Linear Algebra and Appl. 10 (1975), 89-93.

[DU] J. J. Diestel and J. J. Uhl, Jr., Vector measures, Math. Surveys, no. 15, Amer. Math. Soc., Providence, RI, 1977.

[Dix] J. Dixmier, Les algèbres d'opérateurs dans l'espace Hilbertien, Gauthier-Villars, Paris, 1957.

[DF] R. G. Douglas and C. Foias, Infinite dimensional versions of a theorem of Brickman-Fillmore, Indiana Univ. Math. J. 225 (1976), 315-520.

[E] J. Ernest, Charting the operator terrain, Mem. Amer. Math. Soc., No. 171, 1976.

[Fi] P. A. Fillmore, On invariant linear manifolds, Proc. Amer. Math. Soc. 41 (1973), 501-505.

[G] J. Glimm, A Stone-Weierstrass theorem for $C^{*}$-algebras, Ann. of Math. 72 (1960), 216-244.

[Gi] T. A. Gillespie, Boolean algebras of projections and reflexive algebras of operators, Proc. London Math. Soc. (3) 37 (1978), 56-74.

[H1] D. W. Hadwin, An asymptotic double commutant theorem for $C^{*}$-algebras, Trans. Amer. Math. Soc. 244 (1978), 273-297.

[H2] _ Algebraically reflexive linear transformations, Linear and Multilinear Algebra 14 (1983), 225-233.

[H3] _ Approximately reflexive algebras, J. Operator Theory 28 (1992), 51-64.

[H4] _ A reflexivity theorem for subspaces of Calkin algebras, J. Funct. Anal. (to appear).

[HN1] D. W. Hadwin and E. A. Nordgren, Subalgebras of reflexive algebras, J. Operator Theory 7 (1982), 3-23.

[HN2] _ Erratum-subalgebras of reflexive algebras, J. Operator Theory 15 (1986), 203-204.

[HN3] _ Reflexivity and direct sums, Acta Sci. Math. (Szeged) 55 (1991), 181-197.

[HNRR] D. W. Hadwin, E. A. Nordgren, H. Radjavi, and P. Rosenthal, Orbit-reflexive operators, J. London Math. Soc. (2) 34 (1986), 111-119.

[HO] D. W. Hadwin and S.-C. Ong, On algebraic and para-reflexivity, J. Operator Theory 17 (1987), 23-31.

[HOr] D. W. Hadwin and M. Orhon, Reflexivity and approximate reflexivity for Boolean algebras of projections, J. Funct. Anal. 87 (1989), 348-358.

[H] P. R. Halmos, Ten problems in Hilbert space, Bull. Amer. Math. Soc. 76 (1970), 887-933.

[Hi] C. J. Himmelberg, Measurable relations, Fund. Math. 87 (1975), 53-72.

[KL] J. Kraus and D. Larson, Reflexivity and distance formulae, Proc. London Math. Soc. (3) 53 (1986), 340-356.

[La] A. L. Lambert, Strictly cyclic operator algebras, Pacific J. Math. 39 (1971), 717-726.

[L1] D. Larson, Annihilators of operator algebras, Operator Theory: Advances and Applications, Birkhäuser, Berlin, 1982.

[L2] , Hyperreflexivity and a dual product construction, Trans. Amer. Math. Soc. 294 (1986), 79-88.

[L3] - Reflexivity, algebraic reflexivity and linear interpolation, Amer. J. Math. 110 (1988), 283-299.

[LS] A. I. Loginov and V. S. Shulman, On hereditary and intermediate reflexivity of $W^{*}$-algebras, Izv. Akad. Nauk SSSR Ser. Mat. 396 (1975), 1260-1273; Math. USSR Izv. 9 (1975),11891201. (Russian)

[M] W. Mlak, Operator valued representations of function algebras, Linear Operators and Approximation. II (Proc. Conf., Oberwolfach Res. Inst., Oberwolfach, 1974), Internat. Ser. Numer. Math., vol. 25, Birkhäuser, Basel, 1974.

[OT] R. F. Olin and J. E. Thomson, Algebras of subnormal operators, J. Funct. Anal. 37 (1980), 271-301.

[Or] M. Orhon, Locally cyclic representations of $C(K)$, preprint. 
[P] V. I. Paulsen, Completely bounded maps and dilations, Pitman Research Notes in Math., 146, Wiley, New York, 1986.

[Pel] A. Pelczyński, Projections in certain Banach spaces, Studia Math. 19 (1960), 209-228.

[RR] H. Radjavi and P. Rosenthal, Invariant subspaces, Springer-Verlag, New York, Heidelberg, and Berlin, 1973.

[Rd] C. J. Read, The invariant subspace problem for a class of Banach spaces, 2: hypercyclic operators, Israel J. Math. 63 (1988), 1-40.

[RoRo] A. P. Robertson and W. J. Robertson, Topological vector spaces, Cambridge Univ. Press, Cambridge, 1966.

[Ros] S. Rosenoer, Distance estimates for von Neumann algebras, Proc. Amer. Math. Soc. 86 (1982), 248-252.

[S-B] M.-F. Sainte-Beuve, On the extension of von Neumann Aumann's theorem, J. Funct. Anal. 17 (1974), 112-129.

[V] D. Voiculescu, A noncommutative Weyl-von Neumann theorem, Rev. Roumaine Pures Appl. 21 (1976), 97-113.

Department of Mathematics, University of New Hampshire, Durham, New Hampshire 03824

E-mail address: dwhadwin@christa.unh.edu 